\title{
REPRESENTACIÓN DE DOMINIOS TEÓRICOS MEDIANTE RETÍCULOS: EL DOMINIO DE LA HERENCIA BIOLÓGICA DURANTE EL PERIODO 1865-1902*
}

\author{
Diego Méndez y Mario Casanueva \\ UAM-Cuajimalpa (México)
}

\section{Resumen}

En lo que sigue, se reconstruye el dominio de teorías relativas a la herencia biológica, propuestas durante el periodo 1865-1902. Se trata de un episodio científico de mucha controversia y audaces especulaciones, libres de los constreñimientos de un paradigma establecido. En consecuencia, surgieron propuestas muy diversas, para las cuales no empata bien la categoría metateórica de red. De cualquier manera, los elementos teóricos detectados sí guardan vínculos entre sí, debido a que comparten ciertas estructuras, y esto permite introducir mediante un arreglo reticular la idea de dominio, donde la naturaleza de los vínculos no alude a especializaciones de leyes fundamentales sino a la secuencia en el armado de subestructuras. El trabajo explora esta posibilidad combinando el aparato del programa estructuralista con la técnica ordenadora denominada Análisis Formal de Conceptos (Formal Concept Analysis): en concreto se aplica dicho procedimiento a una muestra de veinte reconstrucciones, de elementos teóricos relativos a la herencia entre 1865 y 1902.

Palabras claves: dominio, teorías de la herencia, retículo, análisis formal de conceptos.

\section{Abstract}

This paper reconstructs the domain of biological heredity theories from the period of 1865-1902. Said episode in science was one of much controversy and daring speculations, free of constraints from an established paradigm. Many theoretical proposals were made, the set of which does not fit well with the meta-theoretical category of theory net. No-

Recibido: 12/03/2011. Aceptado: 16/05/2012.

* Este trabajo participa del proyecto de investigación PICTR 2006 N N $^{\circ} 2007$, de la Agencia Nacional de Promoción Científica y Tecnológica de la República Argentina. 
netheless, the theory elements identified are linked to each other, due to the fact that they share certain structures. This allows the introduction of the idea of a theory-domain as a lattice-like arrangement; where links between theory elements do not represent specializations of fundamental laws, but sequences of substructure couplings. The article explores said representation, and does so by combining aspects of the structuralist program's apparatus with the ordination technique of Formal Concept Analysis. In a nutshell, Formal Concept Analysis is applied to twenty reconstructions of theory elements concerning heredity, form the period 1865-1902.

Keywords: domain, heredity theories, lattice, formal concept analysis.

\section{Introducción}

En el último tercio del siglo XIX, varias teorías se disputaban la arena de la herencia: además de las hoy conocidas propuestas de Mendel (pero no tan conocidas en los medios científicos de su época), cabe mencionar la hipótesis darwiniana de la pangénesis, la pangénesis intracelular de de Vries, la ley de herencia ancestral de los biometristas y la teoría de la continuación del germoplasma de Weismann. Otras contribuciones, tales como la propuesta de Brooks y la pangénesis de Galton, también son dignas de mención.

Entre los diversos rasgos atractivos que ofrece la Concepción Estructural, brilla la noción de que las teorías científicas no son entidades aisladas, sino que están vinculadas y forman distintos tipos de alianzas. Al respecto, el programa estructuralista identifica tres clases de ensambles, a saber: redes teóricas, evoluciones teóricas y holones. Existe un cuarto apartado, el marco teórico, propuesto por Moulines (1982) para la termodinámica, pero parece una modalidad tentativa que no ha sido mayormente desarrollada en la literatura estructuralista posterior.

Se antoja pensar que la producción científica tocante a la herencia, de fines del siglo XIX y principios del XX, puede ser asignada a alguna de estas categorías. Ahora bien, semejante colección de teorías no responde a una misma ley fundamental y, en consecuencia, no se adecua a un orden de especialización. Por tanto, el conjunto no parece encajar bien en los rubros de red y evolución teórica. Ciertamente, cabe responder a esto que las propuestas se podrían ubicar en diversas redes teóricas, pero dadas las características del episodio, dichas redes se antojan más como redes en ciernes, con dos o tres elementos, o bien redes degeneradas.

La categoría de marco teórico parece un buen candidato, pero Moulines (1982) señala dos aspectos de ella que no se aplican del todo a nuestro episodio de la herencia. Por una parte, el autor plantea que el marco teórico 
para una determinada clase de teorías se corresponde con una estructura esencial, de tal suerte que todos los armazones conceptuales de las teorías incluidas en el marco - esto es, los ensambles de términos no-T-teóricos y T-teóricos de cada teoría- se pueden mapear a dicha estructura esencial. Además, el aspecto más destacado del marco teórico es que todas las teorías comparten una base operacional. Si bien los esquemas relativos a la herencia del periodo señalado pueden compartir subestructuras - por ejemplo, todas incluyen relaciones de descendencia-, resulta difícil atisbar una estructura esencial que se instancie en todos ellos. Por otro lado, los métodos experimentales privilegiados también son variopintos: algunas propuestas se fincan en técnicas de hibridación y cruzas controladas, otras ponen el acento en la preparación de cortes histológicos y observaciones a través del microscopio, otras más destacan la elaboración de complejos tabulados estadísticos y el estimado de correlaciones. En fin, la categoría de marco teórico no parece englobar bien a bien la gama de teorías sobre la herencia en el último tercio del siglo XIX. Además, se podría discutir que esta categoría está incluida en la clase metacientífica de holón, y quizá por ello el Architectonic, - hasta donde sabemos, la exposición más completa del Programa Estructuralista-, no habla de marcos teóricos.

Parece, entonces, más seguro apostar a que el episodio revela un holón; sin embargo, dicho término abarca grandes porciones de la ciencia, da cabida a disciplinas enteras o trozos extensos de diversas disciplinas -incluso toda la ciencia podría considerarse un holón-, y, en relación a esto, el cuerpo de teorías al que aludimos es algo mucho más acotado.

Los contextos de exploración y controversia, como nuestro capítulo sobre lo hereditario, presentan rasgos distintivos: giran en derredor a un problema o colección acotada de problemas relacionados; involucran científicos de una misma época que, salvo excepciones, están al tanto de la producción intelectual de sus colegas y/o contrincantes; y en tales contextos prolifera la diversidad teórica. Se antoja pensar que ostentan propiedades estructurales especiales y quizá convenga, entonces, estipular una subcategoría para ellos dentro de la clase "holón", que llamaremos "dominio teórico" (en alusión a Shapere, 1977). Para revelar cuáles son esas propiedades especiales, consideramos una buena estrategia examinar y ordenar la variación conceptual subyacente al conjunto de elementos teóricos considerados. Más aún, la tarea de representar tal variabilidad puede verse facilitada haciendo uso de dispositivos formales que, hasta ahora, no figuran en el instrumental del estructuralismo, aunque son completamente compatibles con el enfoque de dicho Programa. En lo que sigue, se exploran estos asuntos. La propuesta a discutir es que los métodos desarrollados por la Concepción Estructural se 
pueden combinar con técnicas del llamado "análisis formal de conceptos" (Formal Concept Analysis), ${ }^{1}$ un desarrollo ligado al campo de la inteligencia artificial, para así destacar las variaciones conceptuales en torno a la herencia, durante el periodo 1865-1902.

Justo es señalar que buena parte del episodio científico aquí privilegiado parece corresponderse con lo que Kuhn denomina ciencia inmadura. Aún no hay un paradigma establecido que dé cuenta sobre el problema de lo hereditario, sino diversas escuelas de pensamiento que lo abordan desde perspectivas distintas, con base en esquemas conceptuales todavía preliminares y muy especulativos. El programa estructuralista se ha orientado más (y con notable éxito) a reconstruir lo que en términos kuhnianos se denomina ciencia madura. No sorprende, entonces, que las nociones de red y evolución teórica, así como la de marco teórico, no se adecuan del todo a un caso de ciencia inmadura, como lo fue el asunto de la herencia entre 1865 y 1902. Pero esto no significa que el estructuralismo deba limitarse a la ciencia madura; el programa es lo suficientemente rico para elaborar las herramientas metateóricas requeridas, en aras de transparentar dinámicas conceptuales de contextos científicos que la propuesta kuhniana ubica en fases de inmadurez. Recientemente, Moulines ha caracterizado cuatro tipos fundamentales de estructuras diacrónicas en la ciencia: cristalización, evolución teórica, incrustación y suplantación con inconmensurabilidad (parcial). En su trabajo, influido por Lorenzano (2006), menciona como un ejemplo plausible de cristalización "la emergencia de la genética 'mendeliana' (1865-1905), o sea, desde los trabajos originales de Mendel hasta el establecimiento del primer 'paradigma' genético por parte de Bateson" (Moulines, 2011). Al igual que Lorenzano y Moulines, consideramos que los trabajos de Mendel, Correns, de Vries, Tchermarck y Bateson pueden incluir un caso de cristalización (el de la genética mendeliana), pero a nosotros nos interesa analizar un panorama más amplio cuya diversidad no es subsumible bajo la idea de cristalización pues, para el periodo analizado, no es posible considerar cristalización alguna, si acaso ello sería un evento posterior.

En realidad, nos parece que en los albores de la genética existe una gran diversidad de escuelas, y de variantes dentro de cada escuela; los casos de mendelianos y biometristas serían tan sólo un par dentro de una gran variedad, cada uno con su propio proceso de cristalización y posterior encuentro. Para Moulines "el proceso de cristalización concluye con el establecimiento

${ }^{1}$ Esta estrategia no es nueva, por ejemplo, la noción estructuralista de aproximación utiliza ideas y métodos topológicos. 
de una red teórica arbórea, es decir, con un solo elemento teórico básico, firmemente establecido" lo cual claramente implica la pérdida de diversidad teórica. Nuestro interés está en el estudio mismo de la variabilidad no de las formas en las que ésta se pierde. Si bien creemos que el presente análisis puede tener puntos de contacto con la cristalización de Moulines, esto habrá que explorarlo en investigaciones subsecuentes.

\section{Concepciones de la herencia entre 1865 y 1902}

La teoría darwiniana de selección natural supone la herencia de variaciones de una generación a la siguiente, pero no abunda en los mecanismos al respecto, ni en la fenomenología de dicha transmisión. Aquí despuntaba un problema digno de investigación, una vez que On the Origin of Species salió de la imprenta. Entre 1865 y 1902 una gama variopinta de especulaciones sobre el tema encontró su lugar en la literatura biológica. Ciertamente, los límites temporales aquí estipulados son obligadamente un tanto arbitrarios, puesto que la herencia era asunto de interés científico antes de 1865 y seguía siéndolo después de 1902. La cota inferior coincide con el reporte de Mendel - tocante a sus experimentos con chícharos y frijolesante la sociedad naturalista de Brünn; el límite superior, 1902, marca el comienzo de lo que los historiadores de la biología han denominado el debate biometrista-mendeliano, con la evaluación que hace Weldon (1901-1902) del trabajo de Mendel y la respuesta de Bateson (1902) a Weldon.

Pudiera parecer que al fijar estos dos linderos temporales, el presente trabajo está orientado a privilegiar el surgimiento de la genética mendeliana, pero este no es el caso. Más bien, el lapso escogido se puede caracterizar como un momento de exploración, suscitado, entre otros, por la publicación del On the Origin of Species. A muy grandes trazos, antes de 1865 el problema de la herencia guarda poca relación con el evolucionismo darwinista, aunque Mendel hace alusión al evolucionismo. ${ }^{2}$ Por otra parte, los desarrollos posteriores a 1902 gravitan hacia la consolidación de un programa de investigación genético, inspirado en el trabajo original

\footnotetext{
${ }^{2}$ Conviene señalar algunas cuestiones acerca del trabajo de Mendel (1866) que lo ponen en una situación un tanto especial. En primer lugar, puede argüirse que el problema principal que quiere destacar el autor, si bien está vinculado con la herencia, es la hibridación: seguir la formación y evolución de los híbridos a través de sus descendientes. Por otra parte, si bien Mendel alude al evolucionismo, lo hace en un contexto hibridista, no darwiniano. Mendel pretende elevar la hibridación de especies como mecanismo evolutivo.
} 
de Mendel, pero no del todo una continuación del mismo. El presente escrito comprende veinte esquemas, que se enlistan en la tabla 1. La muestra es amplia, aunque no agota toda la producción teórica respecto del tema, durante el periodo señalado v.g., las propuestas de Galton (1997) sobre la herencia ancestral o las tesis de Tschermack, de Vries o Correns (estas tres últimas, tradicionalmente consideradas como un "redescubrimiento" de las tesis mendelianas) no han sido consideradas, aunque la muestra suministrada es representativa del dominio bajo estudio.

\begin{tabular}{|c|c|c|c|}
\hline \multicolumn{4}{|c|}{ TABLA 1. ESQUEMAS DE LA HERENCIA Y SIGLAS } \\
\hline B1 & $\begin{array}{l}\text { Bateson (1902): dominancia } \\
\text { completa de alelomorfos }\end{array}$ & M3 & $\begin{array}{l}\text { Mendel (1866): elementos } \\
\text { complejos }\end{array}$ \\
\hline B2 & $\begin{array}{l}\text { Bateson (1902): codominancia de } \\
\text { alelomorfos }\end{array}$ & $\mathrm{P}$ & $\begin{array}{l}\text { Pearson (1896): ley de herencia } \\
\text { ancestral }\end{array}$ \\
\hline B3 & $\begin{array}{l}\text { Bateson (1902): alelomorfos } \\
\text { complejos }\end{array}$ & $\mathrm{V} 1$ & $\begin{array}{l}\text { de Vries (1910 [1889]): pangénesis } \\
\text { intracelular en organismos con } \\
\text { reproducción sexual }\end{array}$ \\
\hline $\mathrm{Br} 1$ & $\begin{array}{l}\text { Brooks (1883): herencia en } \\
\text { organismos con reproducción } \\
\text { sexual }\end{array}$ & $\mathrm{V} 2$ & $\begin{array}{l}\text { de Vries (1910 [1889]): pangénesis } \\
\text { intracelular en organismos con } \\
\text { reproducción sexual y vegetativa }\end{array}$ \\
\hline $\mathrm{Br} 2$ & $\begin{array}{l}\text { Brooks (1883): herencia en } \\
\text { organismos con reproducción por } \\
\text { partenogénesis }\end{array}$ & W1 & $\begin{array}{l}\text { Weismann (1893): continuidad } \\
\text { del germoplasma en organismos } \\
\text { con reproducción sexual }\end{array}$ \\
\hline D1 & $\begin{array}{l}\text { Darwin }(1883) \text { : pangénesis en } \\
\text { organismos con reproducción } \\
\text { sexual }\end{array}$ & W2 & $\begin{array}{l}\text { Weismann (1893): continuidad } \\
\text { del germoplasma en organismos } \\
\text { con reproducción sexual y } \\
\text { vegetativa }\end{array}$ \\
\hline D2 & $\begin{array}{l}\text { Darwin }(1883) \text { : pangénesis en } \\
\text { organismos con reproducción por } \\
\text { partenogénesis }\end{array}$ & W3 & $\begin{array}{l}\text { Weismann (1893): continuidad } \\
\text { del germoplasma en organismos } \\
\text { con reproducción sexual y } \\
\text { alternancia de generaciones }\end{array}$ \\
\hline G & $\begin{array}{l}\text { Galton }(1875) \text { : pangénesis en } \\
\text { organismos con reproducción } \\
\text { sexual }\end{array}$ & W4 & $\begin{array}{l}\text { Weismann (1893): continuidad } \\
\text { del germoplasma en organismos } \\
\text { con reproducción por } \\
\text { partenogénesis }\end{array}$ \\
\hline M1 & $\begin{array}{l}\text { Mendel (1866): dominancia } \\
\text { completa }\end{array}$ & $\mathrm{Y} 1$ & $\begin{array}{l}\text { Yule (1902): dominancia } \\
\text { relativa de alelomorfos }\end{array}$ \\
\hline M2 & Mendel (1866): co-dominancia & $\mathrm{Y} 2$ & $\begin{array}{l}\text { Yule (1902): relación } \\
\text { probabilística entre alelomorfos y } \\
\text { características }\end{array}$ \\
\hline
\end{tabular}




\section{Algunos comentarios sobre las fuentes}

Cabe mencionar que las fuentes de dónde se obtuvieron los elementos de la tabla 1 constituyen los artículos sueltos o libros originales donde el elemento del cual se trate se ofrece en versión preliminar. Esto tiene ciertas implicaciones que es menester señalar. Bien puede ser el caso que lo expresado en la comunicación se ajusta a lo que la Concepción Estructural denomina "modelo efectivo de una teoría (M)" pero igualmente puede ser que sea una hipótesis tentativa y, en consecuencia, no cumpla con algunos requisitos importantes que el estructuralismo reserva para la noción de "modelo efectivo". Por ejemplo, el esquema puede introducir entidades y relaciones que se antoja llamar "teóricas", en tanto que son novedosas y le dan coherencia a la propuesta, pero no abundar sobre métodos empíricos específicos que determinan tales entidades y relaciones. Los términos introducidos son "teóricos" en algún sentido laxo y ambiguo, pero en ausencia de métodos de determinación es difícil saber si estamos o no en presencia de entidades T-teóricas pues el principal criterio para atribuir o negar la T-Teoricidad está directamente relacionado con la forma de determinación.

$\mathrm{Al}$ respecto, la pangénesis de Darwin versa sobre unas entidades orgánicas llamadas gémulas: las células producen gémulas, las gémulas inducen la diferenciación celular, los gametos portan gémulas y toda esta dinámica de gémulas da cuenta de la transmisión de características de progenitores a descendientes. Sin embargo, Darwin no señala qué métodos empíricos precisos ponen de relieve las diversas facetas del comportamiento de las gémulas. Y ningún contemporáneo o sucesor de Darwin parece que lo haya hecho. ${ }^{3}$ Postular la existencia de estas entidades orgánicas, así como de las relaciones que entablan entre sí y con las células, brinda un esquema plausible de la herencia: subordina los informes citológicos, embriológicos e hibridistas que consultó Darwin, pero no deja de ser mera especulación. De hecho, de los trabajos reseñados aquí, sólo los de Mendel, Bateson y Pearson parecen ofrecer métodos claros para determinar las relaciones que introducen, a la vez que son métodos dependientes de la coherencia interna del esquema propuesto (de la ley fundamental).

Otro aspecto interesante acerca de las fuentes, es que pueden presentar varias modalidades de una propuesta teórica, pero tales variantes no están inscritas en una red de especialización. Por ejemplo, Weismann (1893)

${ }^{3}$ Aunque podría considerarse que Galton sí estableció un método para demostrar que las gémulas no circulan por el torrente sanguíneo (véase Gillham, 2001, pp. 173-179). 
desarrolla cuatro versiones de la continuación del germoplasma, para cuatro tipos de organismos que se distinguen por el modo de reproducción. Así, en términos del estructuralismo, las cuatro alternativas de la propuesta weismanniana se montan sobre distintas clases de modelos potenciales parciales y, en consecuencia, ninguna de estas cuatro variantes puede ser una especialización de otra.

\section{Reconstrucción y tabulación de datos}

Antes de seguir permítasenos caracterizar brevemente la noción de dominio teórico. A nuestro parecer, el concepto de dominio enriquece las posibilidades de inteligibilidad de la dinámica de las teorías y sus modelos, pues permite recuperar la variabilidad de respuestas en torno a un problema. Strictu sensu se trata de un tipo particular de holón en donde la relación interteórica que lo caracteriza es la teorización sobre una "subestructura empírica" que, puede ser caracterizada a su vez mediante ciertas "subestructuras parciales". ${ }^{4}$ En otras palabras, un domino teórico es un espacio conceptual que reúne las propuestas alternativas a una pregunta común (o similar) a diferentes modelos dentro de un campo determinado. La cuestón pre-existe a los modelos que la responden, mismos que pueden tener diferentes descripciones y otorgar una importancia distinta a las diversas facetas de la pregunta.

Nos parece que una representación adecuada de un dominio teórico debe poner de relieve los patrones de variación estructural contenidos en él. Se trata entonces de disponer los elementos teóricos involucrados en un arreglo que permita vislumbrar cambios en el ensamblado de entidades y relaciones al pasar de un elemento a otro, siguiendo los caminos o direcciones que plantea el arreglo. Para ello hay múltiples posibilidades, pero la tarea exige antes elaborar un registro de los componentes que corresponden a cada elemento teórico. En el apéndice 1 se muestra una tabla representativa de los esquemas de la herencia (columnas) y las funciones (hileras) que los componen. Dada la gran cantidad de funciones, hemos optado por

\footnotetext{
${ }^{4}$ Un dominio está acotado o bien respecto a un problema, o bien respecto a un tema. Entre dos teorías distintas de un mismo dominio puede no existir ninguna de las relaciones interteóricas actualmente privilegiadas por el estructuralismo. A diferencia de ello, un holón, a secas, puede incluir cualquier relación interteórica con cualquier otra teoría que no necesariamente está incluida en el tema.
} 
subindizarlas, para así no recurrir a demasiados símbolos. Además, todas las funciones con la misma letra griega se refieren a relaciones "del mismo tipo" por ejemplo, $\alpha$ delata funciones tocantes al parentesco (maternidad, paternidad y fraternidad), $\beta$ asuntos concernientes a la progenie, las funciones $\gamma$ inciden en lo relativo a la producción de gametos, esporas y otras células germinales, etc.

Por falta de espacio no podemos presentar las veinte reconstrucciones, ${ }^{5}$ aquí nos limitamos a una de ellas. Hasta donde sabemos, la pangénesis intracelular de de Vries (V1) no ha sido reconstruida en la literatura estructuralista, por lo cual procedemos a ello. Este elemento plantea que los rasgos heredables de los individuos se deben a la existencia de ciertos factores —que de Vries denominó "pangenes"-, los cuales se ubican en los núcleos celulares. Los pangenes migran al citoplasma y ahí configuran la estructura, forma y función de la célula. Dado que la fisonomía del organismo descansa en la organización celular del mismo, los atributos físicos del individuo están determinados, en última instancia, por la actividad de los pangenes. La producción de gametos y la fecundación son los eventos que garantizan la transmisión de estos factores de padres a hijos. Así, el esquema de de Vries consta de tres partes: 1) una tocante a la fecundación; 2) otra relativa a la composición celular de las características físicas y 3) otra más concerniente a la actividad de los pangenes. En los recuadros 1, 2 y 3 mostramos los respectivos predicados conjuntistas y en las figura 1, 2 y 3 ponemos de relieve las respectivas interpretaciones. Cabe señalar que estas figuras están elaboradas a manera de grafos rotulados, pues consideramos que tal formato, a la vez que brinda sentido a los predicados, destaca la estructura relacional de los mismos.

5 Todas las reconstrucciones (a manera de diagramas conjuntistas) están en Méndez (2006). Cabe añadir que a diferencia de las presentaciones canónicas, no hemos separado los axiomas “propios de los "impropios”, pues nos interesa más el análisis de las estructuras conceptuales que de las leyes que circunscriben su funcionamiento. 


\section{Recuadro 1}

$\mathrm{x}$ es un modelo potencial de la fecundación unigamética (FU) sii $\mathrm{x}=\left\langle\mathrm{M}, \mathrm{H}, \mathrm{GM}, \mathrm{GF}, \mathrm{Cig}, \alpha_{1}, \alpha_{4}, \gamma_{1}, \gamma_{2}, \gamma_{3}\right.$, $\left.\gamma_{4}, \delta_{2}, \varepsilon_{1}\right\rangle$ :
0) $\forall \mathrm{x} \in\{\mathrm{M}, \mathrm{H}, \mathrm{GM}, \mathrm{GF}, \mathrm{Cig}\}, 1 \leq|\mathrm{x}| \leq \mathrm{N}$;
3) $\alpha_{4}: \mathrm{M} \cup \mathrm{H} \rightarrow \mathrm{M}$ ( $\alpha_{1}$ es suprayectiva);
1) $\forall \mathrm{x} \in\{\mathrm{M}, \mathrm{H}\}, \forall \mathrm{y} \in\{\mathrm{GM}, \mathrm{GF}\}$ :
4) $\gamma_{1}: \mathbf{M} \rightarrow \mathrm{K}_{0}\left(\gamma_{1}\right.$ es biyectiva $)$;
$\mathrm{x} \cap \mathrm{y}=\varnothing, \mathrm{x} \cap \operatorname{Cig}=\varnothing, \mathrm{y} \cap \operatorname{Cig}=\varnothing ;$
5) $\kappa_{2}: \mathrm{H} \rightarrow \mathrm{K}\left(\gamma_{2}\right.$ es biyectiva);
Def. 1) $\mathrm{K}_{0} \subset \operatorname{Pot}(\mathrm{GM})$;
6) $\gamma_{3}: \mathrm{GM} \rightarrow \mathrm{K}_{0}$ ( $\gamma_{3}$ es suprayectiva);
Def. 2) $\mathrm{K} \subset \operatorname{Pot}(\mathrm{GF})$;
7) $\gamma_{4}: \mathrm{GF} \rightarrow \mathrm{K}\left(\gamma_{4}\right.$ es suprayectiva $)$;
Def. 3) $\mathrm{PG} \subset \mathrm{GM} \times \mathrm{GF}$
8) $\delta_{2}: \mathrm{PG} \rightarrow \mathrm{Cig}\left(\delta_{2}\right.$ es biyectiva);
2) $\alpha_{1}: \mathrm{M} \cup \mathrm{H} \rightarrow \mathrm{H}$ ( $\alpha_{1}$ es suprayectiva);
9) $\varepsilon_{1}:(\mathrm{M} \cup \mathrm{H}) \rightarrow \mathrm{Cig}$ ( $\varepsilon_{1}$ es suprayectiva).
$\mathrm{x}$ es un modelo actual de FUsii:
0) $\mathrm{x}$ es un modelo potencial de FU;
1) $\forall \mathrm{x} \in \mathrm{M} \cup \mathrm{H}, \alpha_{1}(\mathrm{x})=\gamma_{2}^{-1} \circ \gamma_{4} \circ \pi_{2} \circ \delta_{2}{ }^{-1} \circ \varepsilon_{1}(\mathrm{x}) \wedge \alpha_{4}(\mathrm{x})=\gamma_{1}^{-1} \circ \gamma_{3} \circ \pi_{1} \circ \delta_{2}{ }^{-1} \circ \varepsilon_{1}(\mathrm{x})$.

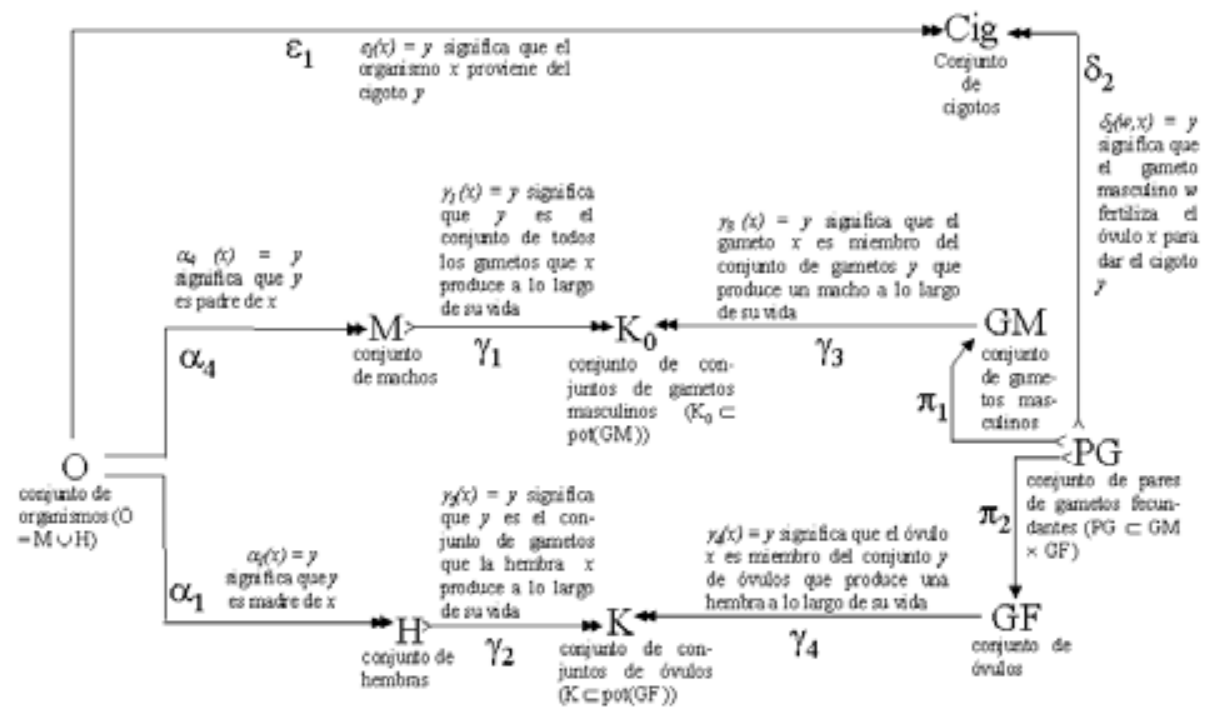

Ley fundamental de FU: $\forall \mathrm{x} \in \mathrm{O}, \alpha_{1}(\mathrm{x})=\gamma_{2}^{-1} \circ \gamma_{4} \circ \pi_{2} \circ \delta_{2}{ }^{-1} \otimes \varepsilon_{1}(\mathrm{x}) \wedge \alpha_{4}(\mathrm{x})=\gamma_{1}^{-1} \circ \gamma_{3} \circ \pi_{1} \circ \delta_{2}{ }^{-1} \circ \varepsilon_{1}(\mathrm{x})$

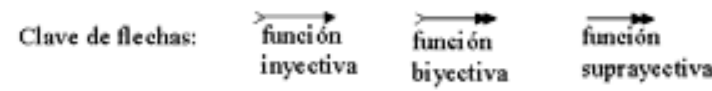

Figura 1. Fecundación unigamética (FU) 


\section{Recuadro 2}

$x$ es un modelo potencial de "características como mosaicos celulares" (CMC) sii $x=\left\langle\mathrm{O}, \mathrm{Ec}, \mathrm{Car}_{1}, \mathrm{Car}_{2}, \ldots\right.$ $\left.\operatorname{Car}_{p} \mathrm{C}_{1}, \mathrm{C}_{2}, \ldots, \mathrm{C}_{\mathrm{n}}, \varepsilon_{6}, \zeta_{1}, \eta_{1}, \eta_{2}, \eta_{2}, \eta_{3}, \eta_{3}, \eta_{4}, \eta_{5}, \eta_{6}\right\rangle:$

0) $\forall \mathrm{x} \in\left\{\mathrm{O}, \mathrm{Ee}, \mathrm{Car}_{1}, \ldots, \mathrm{Car}_{\mathrm{p}}, \mathrm{C}_{1}, \ldots, \mathrm{C}_{\mathrm{n}}\right\}, 1 \leq|\mathrm{x}| \mathrm{s}$ N;

2) $\varepsilon_{6}: \mathrm{O} \times \mathrm{Ee} \rightarrow \mathrm{O} \times \mathrm{Ec}$ ( $\varepsilon_{6}$ es suprayectiva)

1) $\forall x, y \in\left\{O, E e, C a r_{1}, \ldots, C a r_{p} C_{1}, \ldots, C_{n}\right\}, x \neq y:$ $\mathrm{x} \cap \mathrm{y}=\varnothing$;

Def. 1) Dsp $\subset\left\{z \mid z \in \Pi_{i \in h} \operatorname{Car}_{i} \wedge A \subset\{1, \ldots, q\}\right\}$;

Def. 2) MC $\subset \operatorname{Pot}\left(\cup_{3} \mathrm{C}_{2}, 1 \leq \mathrm{i} \leq \mathrm{n}\right)$;

Def. 3) EC $\subset \operatorname{Pot}\left(\cup_{3} C_{p}, 1 \leq \mathrm{i} \leq \mathrm{n}\right), \mathrm{EC} \neq \mathrm{MC}$;

Def. 4) SCC $\subset\left\{z \mid z=\left\langle z_{1}, z_{2}, \ldots, z_{k, 21}\right\rangle \wedge z_{1 \leq k} \in\right.$ $\mathrm{MC}$;

Def 5) $C^{*} \subset\left\{z \mid\left(z=\left\langle z_{1}, z_{2}, \ldots, z_{k \geq 1}\right\rangle \wedge z_{i \leq k} \in\right.\right.$ $\left.\left.\left.\cup_{i} C_{i}(1 \leq i \leq n)\right) \cup z \in \cup_{i} C_{i}(1 \leq i \leq n)\right)\right\}$

3) $\eta_{1} \subset \mathrm{O} \times \mathrm{Ee} \times \mathrm{EC}$;

4) $\eta_{2}: \mathrm{SCC} \rightarrow$ Dsp ( $\eta_{2}$ es suprayeetiva);

5) $\eta_{2 *}: \mathrm{EC} \rightarrow \operatorname{SCC}\left(\eta_{2}+\right.$ es biyectiva);

6) $\eta_{3} \subset \mathrm{MC} \times \cup_{1} \operatorname{Car}_{4}(1 \leq \mathrm{i} \leq \mathrm{p})$;

7) $\eta_{4}: \mathrm{EC} \rightarrow \mathrm{EC}\left(\eta_{4}\right.$ es suprayectiva);

8) $\eta_{5}: \cup_{i} C_{i}(1 \leq \mathrm{i} \leq \mathrm{n}) \rightarrow \mathrm{C}^{*}\left(\eta_{y^{*}}\right.$ es biyectiva);

9) $\eta_{6}: \cup_{1} C_{1}(1 \leq \mathrm{i} \leq \mathrm{n}) \times \mathrm{C}^{*} \times \cup_{\mathrm{i}} \mathrm{C}_{\mathrm{i}}(1 \leq \mathrm{i} \leq \mathrm{n}) \rightarrow$ $\mathrm{EC} \times \mathrm{EC}$

9.1) $\operatorname{si} \eta_{6}(\langle r, s, t\rangle)=\langle x, y\rangle$ entonees $s=\eta_{5}(r)$ ᄉ existe una $i$ tal que $\mathrm{t}=\pi_{3}(\mathrm{~s}) \wedge \mathrm{y}=\eta_{4}(\mathrm{x})$

$\mathrm{x}$ es un modelo actual de CMC sii:

0) $x$ es un modelo potencial de CMC;

1) $\forall x \in O \times$ Ec: i) $\zeta_{1}(x)=\eta_{2} \eta_{2 *}(y)$ sii $\langle x, y\rangle \in \eta_{1} ;$ ii) si $\pi_{i} \zeta_{1}(x)=y$ entonces existe una $z \in E C$ tal que $\langle x$, $z\rangle \in \eta_{1} \cap\left\langle\pi_{1} \eta_{2} \eta_{2}(z), y\right\rangle \in \eta_{3} ;$ iii) $\forall\langle x, y\rangle \in \eta_{1}$ : iii.a) existe un número natural $k$ tal que $\left\langle\varepsilon_{6}(x), \eta_{4}{ }^{k}(y)\right\rangle \in$ $\eta_{1}$; iii.b) $\eta_{4}(\mathrm{y}) \neq \mathrm{y}$ sii existe una $w \in \cup_{1} \mathrm{C}_{\mathrm{i}}(1 \leq \mathrm{i} \leq \mathrm{n})$ tal que $\mathrm{w} \in \mathrm{y} \wedge$ para alguna $i: \pi_{1} \eta_{5}(\mathrm{w}) \notin \mathrm{y} \wedge \pi_{1} \eta_{5}(\mathrm{w})$ $\in \eta_{d}(y)$.

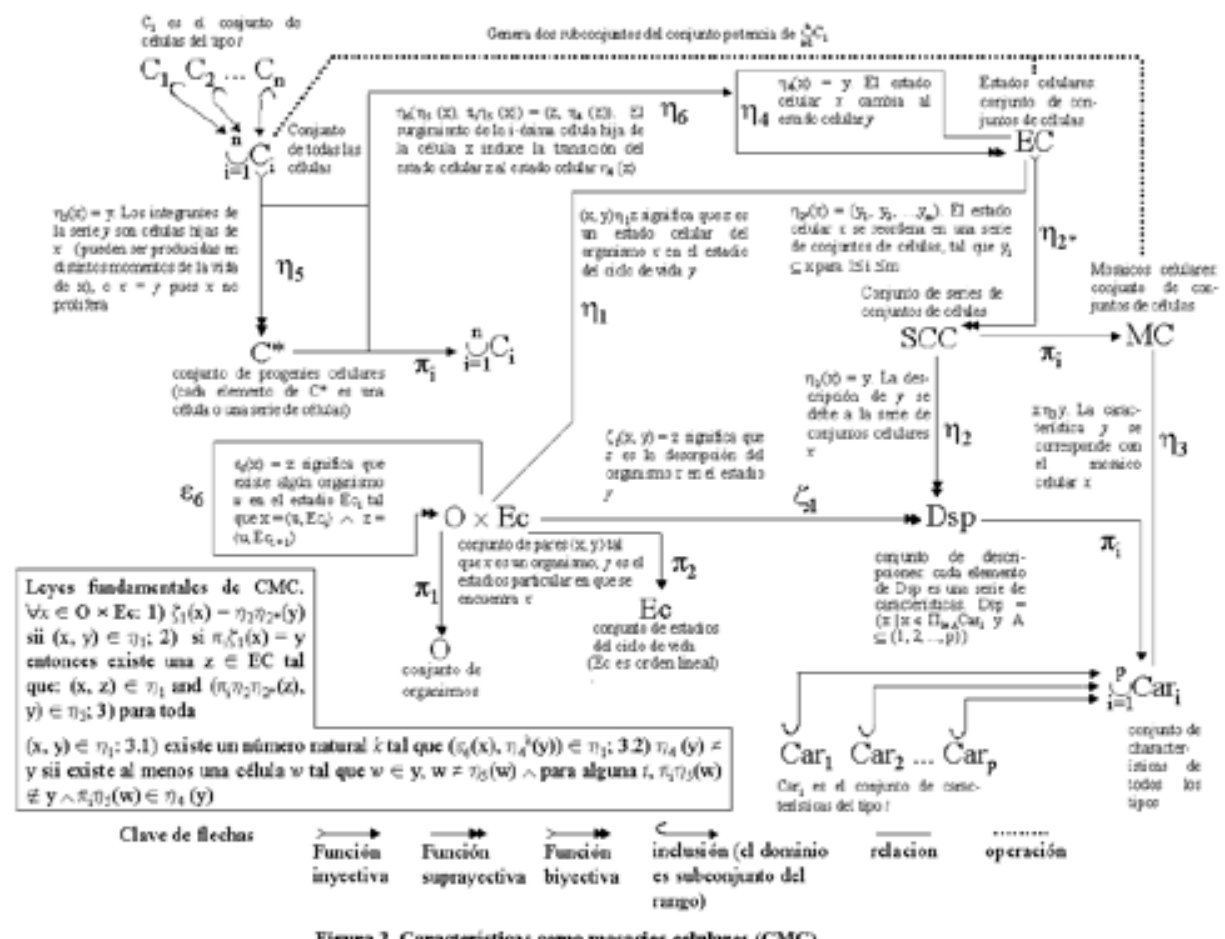

Figura 2. Caracteristicas como mosades celalares (CMC) 


\section{Recuadro 3}

$x$ es un modelo potencial de la "pangénesis intracelular de De Vires para organismos con reproducción sexual" (V1) sii $\mathrm{x}=\left\langle\mathrm{M}, \mathrm{H}, \mathrm{GM}, \mathrm{GF}, \mathrm{Cig}, \mathrm{O}, \mathrm{Ec}, \mathrm{Car}_{1}, \mathrm{Car}_{2}, \ldots \mathrm{Car}_{\mathrm{p}} \mathrm{C}_{1}, \mathrm{C}_{2}, \ldots, \mathrm{C}_{\mathrm{n}}, \mathrm{Og}_{1}, \mathrm{O}_{2}, \ldots, \mathrm{Og}_{\mathrm{r}} \mathrm{N}\right.$, $\left.\mathrm{Fh}_{1}, \mathrm{Fh}_{2}, \ldots, \mathrm{Fh}_{\mathrm{h}}, \alpha_{1}, \alpha_{4}, \gamma_{1}, \gamma_{2}, \gamma_{3}, \gamma_{4}, \delta_{2}, s_{1}, \delta_{6}, \zeta_{1}, \eta_{1}, \eta_{2}, \eta_{2^{*}}, \eta_{3}, \eta_{3}, \eta_{4}, \eta_{5}, \eta_{6}, \theta_{4}, z_{5}, 2_{6}, \lambda_{4}, \lambda_{5}, \xi_{2}, o_{1}, o_{2}\right\rangle:$

$0) \mathrm{x}^{*}=\left\langle\mathrm{M}, \mathrm{H}, \mathrm{GM}, \mathrm{GF}, \mathrm{Cig}, \alpha_{1}, \alpha_{4}, \gamma_{1}, \gamma_{2}, \gamma_{3}, \gamma_{4}, \delta_{2}\right.$, $\left.s_{1}\right\rangle \in \mathrm{M}(\mathrm{FU})$

1) $\mathrm{x}^{* *}=\left\langle\mathrm{O}, \mathrm{Es}, \mathrm{Car}_{1}, \mathrm{Car}_{2}, \ldots \mathrm{Car}_{\mathrm{p}}, \mathrm{C}_{1}, \mathrm{C}_{2}, \ldots, \mathrm{C}_{\mathrm{n}}\right.$, $\left.\varepsilon_{6}, \zeta_{1}, \eta_{1}, \eta_{2}, \eta_{2}, \eta_{3}, \eta_{3}, \eta_{4}, \eta_{5}, \eta_{6}\right\rangle \in \mathrm{M}(\mathrm{CMC})$

1.1) $\mathrm{O}=\mathrm{M} \cup \mathrm{H}$

2) $\forall x \in\left\{\mathrm{Og}_{1}, \mathrm{Og}_{2}, \ldots, \mathrm{Og}_{\mathrm{r}} \mathrm{N}, \mathrm{Fh}_{1}, \mathrm{Fh}_{2}, \ldots, \mathrm{Fh}_{\mathrm{y}}\right\}$, $1 \leq|\mathrm{x}| \leq \mathrm{N}$;

3) $\forall x, y \in\left\{O_{g_{1}}, O_{g_{2}}, \ldots, O_{g_{t}} N, F_{1}, F_{2}, \ldots, F_{h}\right\}$, $\mathrm{x} \neq \mathrm{y}, \mathrm{x} \cap \mathrm{y}=\varnothing$;

Nota 1) $\forall x \in O g_{i}, x=\left\langle z_{1} y_{1}, \ldots z_{1} y_{w}\right\rangle$ tal que $z_{j} \in$ $\cup_{i(1 \leq i \leq q} \mathbf{F h}_{i} \wedge y_{j(1 \leq i \leq w)} \subset \mathbf{R}^{3} \wedge y_{1} \cup y_{2}, \ldots, \cup y_{0}$ es una región conexa;

6) 2. $\mathrm{FFh}^{2} \rightarrow \mathrm{FFh}\left(2_{6}\right.$ depende de $\left.z_{5}\right)$;

7) $\xi_{2}: U_{i(1 \leq i \leq q)} F h_{i} \rightarrow\left(U_{i(1 \leq i \leq q)} F h_{i}\right)^{2}$;

8) $\lambda_{4}: F_{4} \times U_{i(1 \leq i \leq x)} O_{g_{i}} \rightarrow U_{i(1 \leq i \leq t)} O_{g_{i}} \lambda_{4}$ es parcial)

8.1) si $\lambda_{4}(\langle x, y\rangle)=z$ entonees existe una $w \in F F h$ tal que $x=\pi_{2} \xi_{2}\left(w^{*}\right) \wedge w^{*} \in \pi_{1}(w) ;$

8.2) si $\lambda_{4}(\langle x, y\rangle)=z \wedge y \in O g_{j}$, entonces $z \notin O g_{i}$;

9) $\lambda_{5}: \mathrm{Fh}_{\mathrm{i}} \times U_{\mathrm{i}(1 \leq \mathrm{i} \leq \mathrm{i})} \mathrm{Og}_{\mathrm{i}} \times U_{\mathrm{i}(1 \leq \mathrm{i} \leq \mathrm{i})} \mathrm{Og}_{\mathrm{i}} \rightarrow$

$\mathrm{EC} \times \mathrm{EC}$

9.1) si $\lambda_{5}(\langle x, y, z\rangle)=\langle u, v\rangle$ entonees $z=\lambda_{4}(\langle x, y\rangle)$ $\wedge \mathrm{v}=\eta_{4}(\mathrm{u})$

Def. 1) FFh $\subset\left\{z \mid z=\left\langle z_{1}, z_{2}, \ldots, z_{y}\right\rangle\right.$ tal que $z_{1} \in$ $F h_{1} \cup z_{1} \in F h_{1}^{k}$ para alguna $\left.k \geq 2\right\}$;

10) $O_{1}: U_{i(15 i s y)} O_{g_{i}} \rightarrow U_{i(15 i s a)} C_{i}\left(O_{1}\right.$ es suprayeetiva);

4) $\theta_{4}: \mathrm{N} \rightarrow \mathrm{FFh}\left(\theta_{4}\right.$ cs biyectiva);

11) $o_{2}: U_{i(1 \leq i \leq n)} C_{1} \rightarrow \mathrm{N}\left(O_{2}\right.$ es biyectiva);

5) $z_{5}: U_{i(15 i \leq q)} \mathbf{F h}{ }_{1}^{2} \rightarrow U_{i(1 \leq i s q)} F_{h_{1}}$;

\section{Recuadro 3 (continuación)}

$x$ es un modelo actual de la "pangénesis intracelular de De Vires para organismos con reprodueción sexual" (V1) sii :

0) $x$ es un modelo potencial de V1

1) $\forall z \in O \times E s, \exists l w \in \gamma_{1} \alpha_{4} \pi_{1}(z), \exists t w^{*} \in \gamma_{2} \alpha_{1} \pi_{1}(z):$

i) Si $\varepsilon_{1} \pi_{1}(z)=\delta_{2}\left\langle w, w^{*}\right\rangle$ entonces $\theta_{4} O_{2} \varepsilon_{1} \pi_{1}(z)=2\left(\left\langle\theta_{4} O_{2} w, \theta_{4} O_{2} w^{*}\right\rangle\right)$

2) $\forall z \in O \times E c, \forall y \in E C, \exists u \in U_{i(1 \leq i \leq n)} C_{i}$ :

i) Si z $\eta_{1} y \wedge u \in y \wedge y \neq\left\{\theta_{4} O_{2} \varepsilon_{1} \pi_{1}(z)\right\}$ entonces $\forall i \in\{1,2, \ldots q\}, \forall x^{*} \hat{\epsilon} \pi_{i} \theta_{4} O_{2}(u), \exists x \in \hat{\epsilon} \pi_{i} \theta_{4} O_{2} S_{1} \pi_{1}(z)$, $\exists k \in \mathbb{N}, \exists\left\langle a_{1}, a_{2}, \ldots, a_{k}\right\rangle \in\{1,2\}^{k}$ tal que $\left.\left.x^{*}=\pi_{2}, \xi_{2}, \ldots \pi_{2} \xi_{2} \pi_{a 1} \xi_{2}(x)\right)\right]$

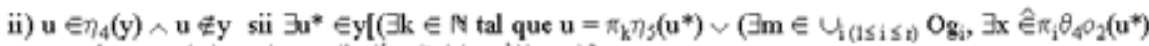
tal que $\left.\left.o_{1}(\mathbf{m})=\mathbf{u}^{*} \wedge O_{1}\left(\lambda_{4}\left(\left\langle\pi_{2} \xi_{2}(\mathbf{x}), \mathbf{m}\right\rangle\right)\right)=\mathbf{u}\right)\right]$ 


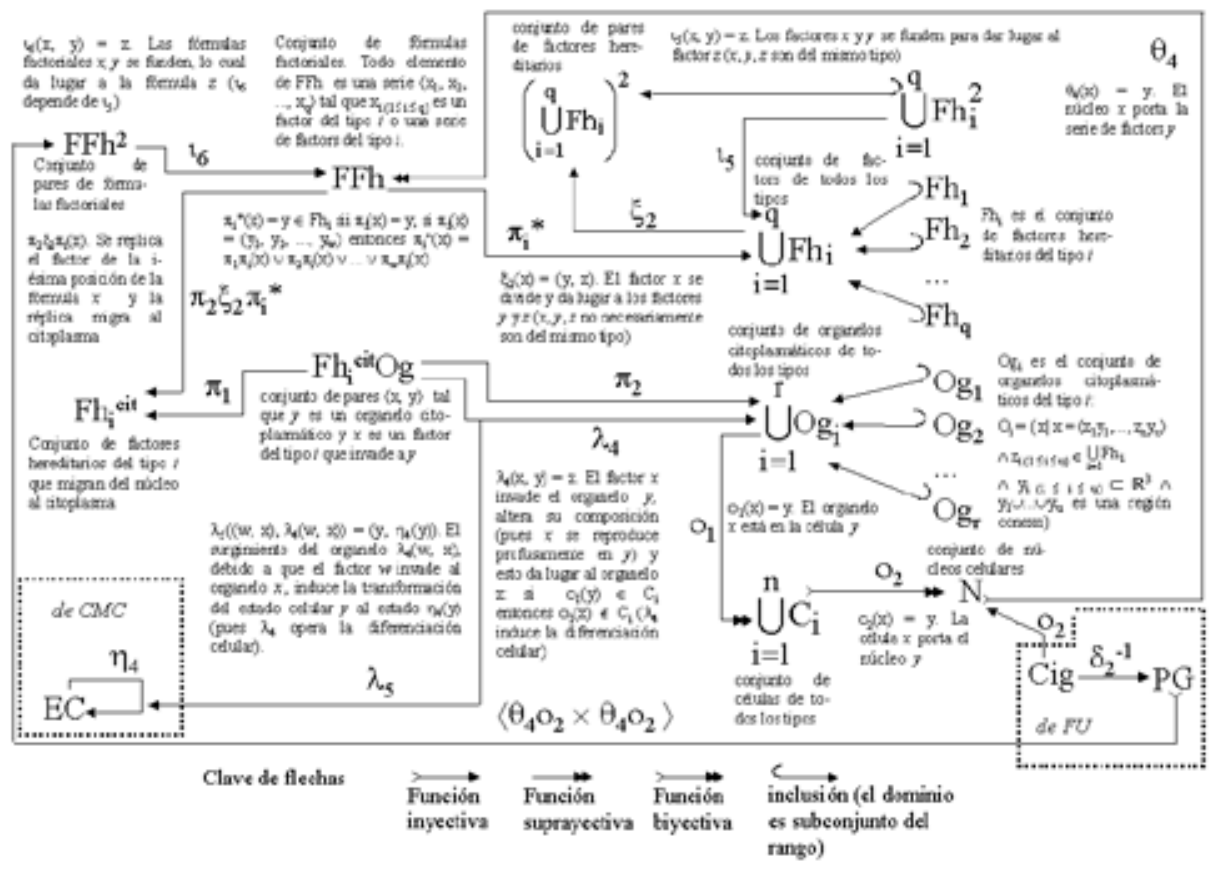

Figura 3. Supraestructura de la pangénesis intracelular de De Vries

\section{Breve explicación del "análisis formal de conceptos"}

Puesto que el "análisis formal de conceptos" (Formal Concept Analysis) se finca en la teoría de retículos, conviene dar una muy breve semblanza de tales objetos matemáticos. Un retículo es un conjunto parcialmente ordenado (sujeto a una relación reflexiva, antisimétrica y transitiva) de tal suerte que para cualesquiera dos elementos del conjunto existe una mínima cota superior y una máxima cota inferior. También se caracteriza por dos operaciones binarias, que en inglés se denominan meet y join. Las propiedades de estas operaciones, así como sus vínculos con la relación de orden, se muestran en el recuadro 4. Ahí también se ofrece un ejemplar, en el cual se ilustra una instancia de join y meet, y, además, se presenta un caso de orden que no es un retículo. 


\section{Recuadro 4}

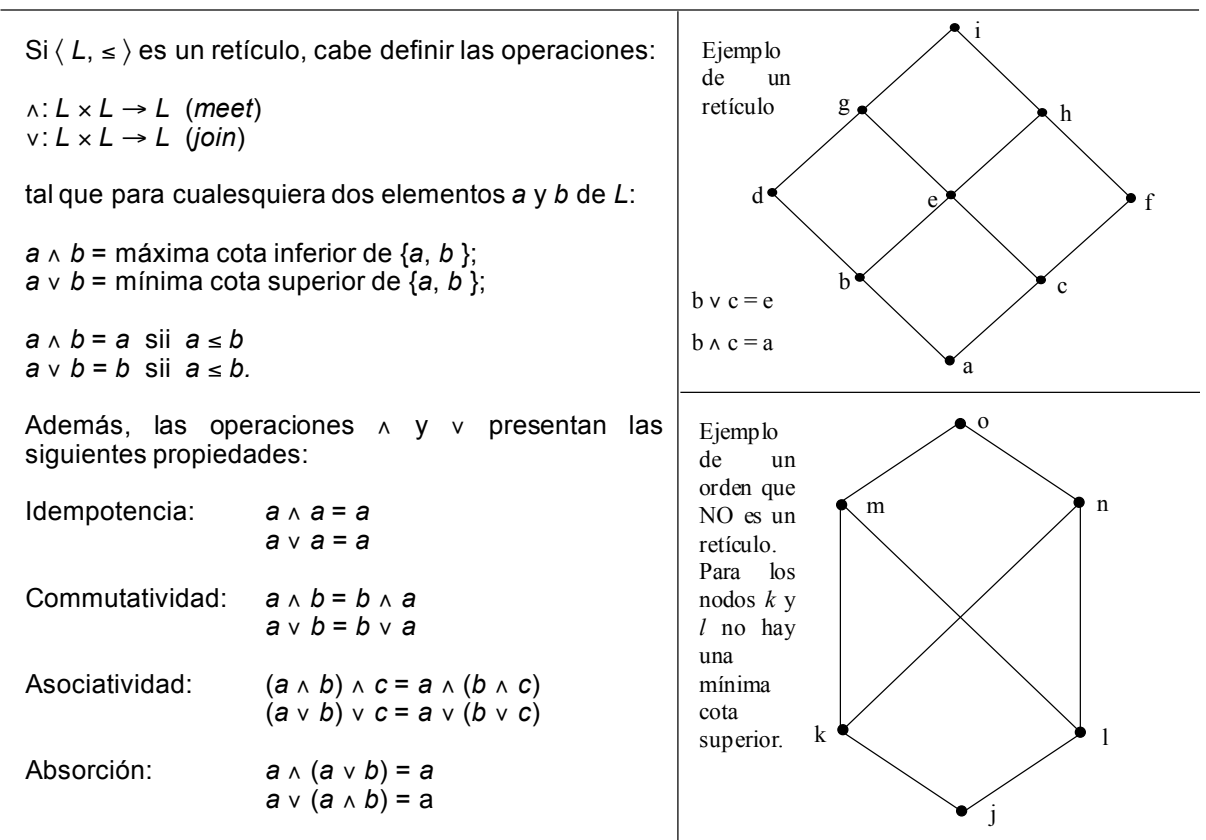

Recuadro 5

\begin{tabular}{|c|c|}
\hline Contexto formal: & $\begin{array}{l}\mathrm{K}:=(\mathrm{G}, \mathrm{M}, \mathrm{I}) \text { tal que } \mathrm{G} \text { es un conjunto de objetos, } \mathrm{M} \text { es un conjunto de } \\
\begin{array}{l}\mathrm{g}, \mathrm{m}) \in \mathrm{I} \text { indica } \\
\mathrm{m} .\end{array}\end{array}$ \\
\hline $\begin{array}{l}\text { Operador *: } \\
\text { Concepto formal: }\end{array}$ & $\begin{array}{l}\text { Para cualesquier } X \subseteq G \text { y } Y \subseteq M: \\
X \rightarrow X^{*}:=\{m \in M \mid g l m \text { para toda } g \in X\} \\
Y \rightarrow Y^{*}:=\{g \in G \mid \text { glm para toda } m \in Y\} \text {. } \\
\text { El operador }{ }^{*} \text { satisfacen las siguientes condiciones: } \\
\text { 1) } Z_{1} \subseteq Z_{2} \Rightarrow Z_{1}^{*} \supseteq Z_{2}^{*}\left(\text { sea que } Z_{1}, Z_{2} \subseteq G \circ Z_{1}, Z_{2} \subseteq M\right) \\
\left.\text { 2) } Z \subseteq Z^{* *} \text { (sea que } Z \subseteq G \circ Z \subseteq M\right) \\
\left.\text { 3) } Z^{* * *}=Z^{*} \text { (sea que } Z \subseteq G \circ Z \subseteq M\right) \\
(X, Y) \text { es un concepto formal del contexto formal }(G, M, l) \text { sii: } \\
X \subseteq G, Y \subseteq M, X=Y^{*} \text { y } Y=X^{*}\left(\text { lo cual implica que } X=X^{* *}\right)\end{array}$ \\
\hline Relación de orden subconcepto-s & 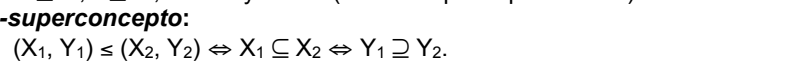 \\
\hline 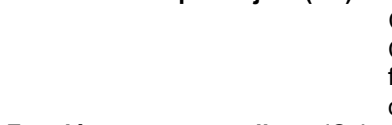 & $\begin{array}{l}\text { Co: } G \rightarrow \operatorname{Pot}(G) \times \text { Pot }(M) \text { tal que para todo } g \in G, C o(g)=\left(\{g\}^{* *},\{g\}^{*}\right) \text {. } \\
\text { Co(g) es el concepto-objeto de } g \text { y } \operatorname{Co}(g) \leq\left(A, A^{*}\right) \text { para todo concepto } \\
\text { formal }(A, \\
\text { donde } g \in A\end{array}$ \\
\hline Función concepto-atributo (Ca): & $\begin{array}{l}\text { Ca: } M \rightarrow \operatorname{Pot}(G) \times \operatorname{Pot}(M) \text { tal que para todo } m \in M, C a(m)=\left(\{m\}^{*},\right. \\
\left.\{m\}^{* *}\right) \text {. Ca }(m) \text { es el concepto-atributo de } m \text { y }\left(A, A^{*}\right) \leq C a(m) \text { para todo } \\
\text { concepto }\left(A, A^{*}\right) \text { donde } \quad m \in A^{*} \text {. }\end{array}$ \\
\hline
\end{tabular}


Bien, el análisis formal de conceptos parte de la idea de que los conceptos están constituidos por sus extensiones e intensiones, pero junto a esto plantea una serie de estipulaciones adicionales que se resumen en el recuadro 5. Dado el concepto formal representado por el par $(X, Y)$, el conjunto $X$ se corresponde con la extensión (relativa al contexto) e $Y$ con la intensión. Así, $(X, Y)$ se corresponde con el concepto relativo a las cosas que tienen las propiedades de $Y$. Con base en todas estas indicaciones, cabe representar la colección de conceptos derivados de un contexto formal como un retículo.

En aras de fijar ideas, conviene recurrir a un ejemplo hipotético, cuyo contexto formal se presenta en la tabla 2 (las letras latinas mayúsculas corresponden a objetos, las letras griegas minúsculas a atributos). La figura 4 muestra el retículo derivada de este tabulado. Las líneas entre nodos indican la relación de orden, superconcepto-subconcepto, antes mencionada. El nodo superior corresponde a $(\{\mathrm{A}, \mathrm{B}, \mathrm{C}, \mathrm{D}, \mathrm{E}\},\{\alpha\})$ de modo que todos los elementos de $\{\mathrm{A}, \mathrm{B}, \mathrm{C}, \mathrm{D}, \mathrm{E}\}$ comparten el atributo $\alpha$, o puesto con los operadores de arriba, $\{\mathrm{A}, \mathrm{B}, \mathrm{C}, \mathrm{D}, \mathrm{E}\}^{*}=\{\alpha\}$ y $\{\alpha\}^{*}=\{\mathrm{A}, \mathrm{B}, \mathrm{C}, \mathrm{D}, \mathrm{E}\}$. En cambio, el nodo inferior se corresponde con $(\varnothing,\{\alpha, \beta, \gamma, \ldots, v\})$ puesto que ningún objeto de la tabla presenta todos los atributos. Nótese que no todo subconjunto de objetos ni todo subconjunto de atributos está representado en el arreglo; solamente figuran aquellos que cumplen con lo estipulado en el recuadro 5. Por ejemplo, el conjunto $\{B, D\}$ no es representativo de ningún nodo del retículo, pues los objetos $\mathrm{B}$ y $\mathrm{D}$ sólo comparten el atributo $\alpha$, pero en relación al contexto de la tabla 2 , la extensión del concepto concerniente a las cosas que tienen la propiedad $\alpha$ incluye todos los objetos del tabulado. Por otra parte, el conjunto $\{\beta, \mu\}$ tampoco es representativo de un nodo, pues la tabla 2 no presenta objeto alguno que tenga ambos atributos a la vez. Es decir, del contexto no se desprende un concepto indicativo de las cosas que tienen las propiedades $\beta$ y $\mu$, salvo el concepto absurdo con extensión vacía, representado por el punto inferior del retículo.

\begin{tabular}{|l|l|l|l|l|l|l|l|l|l|l|l|l|l|l|l|l|l|l|l|l|}
\hline \multicolumn{11}{|c|}{ TABLA 2. CONTEXTO FORMAL HIPOTÉTICO } \\
\hline & $\alpha$ & $\beta$ & $\gamma$ & $\delta$ & $\varepsilon$ & $\zeta$ & $\eta$ & $\theta$ & $\iota$ & $\kappa$ & $\lambda$ & $\mu$ & $\nu$ & $\xi$ & o & $\pi$ & $\rho$ & $\sigma$ & $\tau$ & $v$ \\
\hline $\mathrm{A}$ & $\mathrm{X}$ & $\mathrm{X}$ & & & & $\mathrm{X}$ & & & & & $\mathrm{X}$ & & & $\mathrm{X}$ & & $\mathrm{X}$ & & & & \\
\hline $\mathrm{B}$ & $\mathrm{X}$ & & $\mathrm{X}$ & & & & $\mathrm{X}$ & & & & & $\mathrm{X}$ & & & $\mathrm{X}$ & & $\mathrm{X}$ & & & \\
\hline $\mathrm{C}$ & $\mathrm{X}$ & $\mathrm{X}$ & & & & $\mathrm{X}$ & & & & & & & $\mathrm{X}$ & $\mathrm{X}$ & & & & $\mathrm{X}$ & & \\
\hline $\mathrm{D}$ & $\mathrm{X}$ & & & $\mathrm{X}$ & & & & $\mathrm{X}$ & $\mathrm{X}$ & & $\mathrm{X}$ & & & $\mathrm{X}$ & & & & & $\mathrm{X}$ & \\
\hline $\mathrm{E}$ & $\mathrm{X}$ & & & & $\mathrm{X}$ & & & $\mathrm{X}$ & & $\mathrm{X}$ & & $\mathrm{X}$ & & & $\mathrm{X}$ & & & & & $\mathrm{X}$ \\
\hline
\end{tabular}




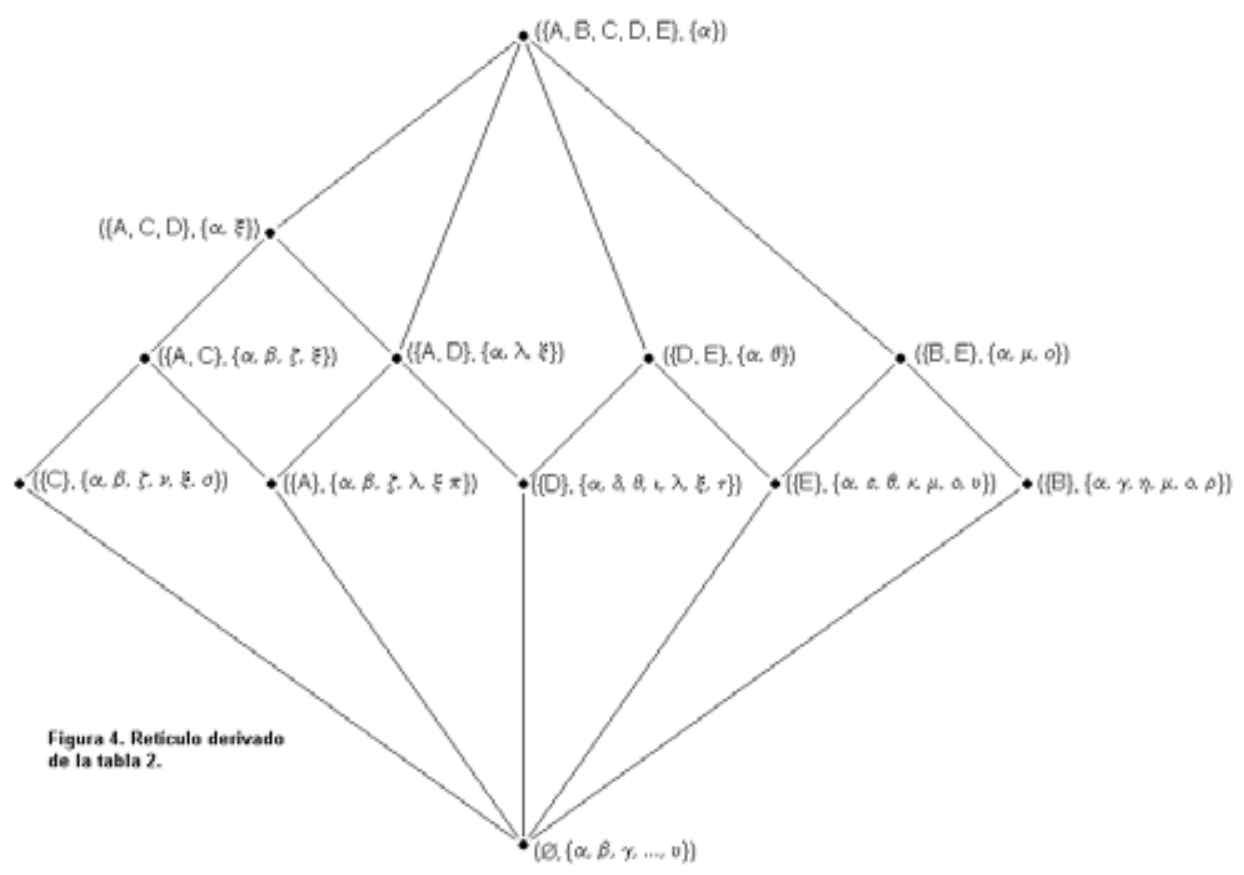

Arriba del nodo inferior se ubican los conceptos-objeto de cada objeto de la tabla. En este caso, a cada objeto le corresponde un concepto-objeto distinto, pero si el tabulado fuera de tal manera que dos objetos presentan idénticos atributos, a ambos les correspondería el mismo concepto-objeto. Para cualquier nodo (X, Y) entre el máximo superior y la serie (anticadena) de concepto-objetos, $\mathrm{Y}$ incluye aquellos atributos que figuran en los nodos ubicados en caminos que parten de $(\mathrm{X}, \mathrm{Y})$ y ascienden al nodo superior. Puede ocurrir que el nodo (X, Y) se corresponda con el concepto-atributo de uno o más atributos, por lo cual, estos últimos también se incluyen en Y. En cambio, el conjunto de objetos $\mathrm{X}$ es la unión de aquellos conjuntos de objetos pertenecientes a nodos ubicados en caminos que parten de $(\mathrm{X}, \mathrm{Y})$ y corren en dirección descendiente. Dadas estas reglas de lectura, la figura 4 se puede simplificar sólo rotulando los nodos representativos de conceptosobjetos y conceptos-atributos con las letras correspondientes. Esto se hace en la figura 5 y, como puede verse, cabe la posibilidad de que un conceptoobjeto también sea un concepto-atributo. 


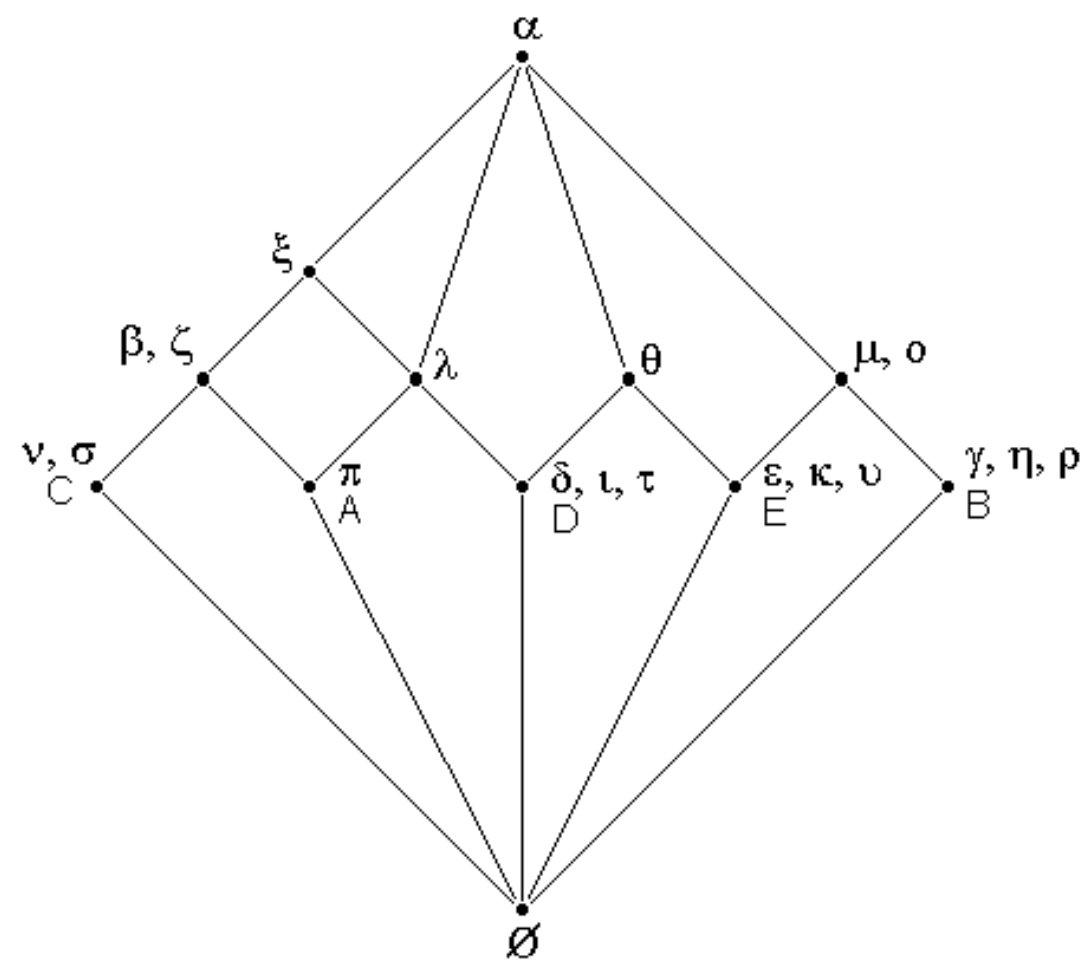

Figura 5. Retículo derivado de la tabla 2 (sólo se indican conceptos-objeto y concepto-atributo)

Hay otro asunto que vale la pena destacar: a partir de los retículos de las figuras 4 y 5: se puede inferir una tendencia de cambio estructural en relación a la serie $\mathrm{C}-\mathrm{A}-\mathrm{D}-\mathrm{E}-\mathrm{B}$, como se expone en la figura 6. Ciertamente, esto es una cuestión colateral, pues al fin al cabo el orden que manifiesta el retículo es vertical (y de hecho, es en dos sentidos: arriba-abajo y abajo-arriba); se preserva si, en desdén a la estética, dispusiéramos los nodos de otra manera en sentido horizontal. Generaríamos un diagrama más abigarrado, con algunos cruces de líneas, pero el orden constitutivo del retículo sería el mismo. No obstante, los libros introductorios a la teoría de retículos — v.g. Grätzer (2000) — señalan conveniente que los diagramas representativos de estos ordenes sean óptimos, esto es, diagramas con el menor cruce posible de líneas. Nuestra apreciación de semejante recomendación es que los diagramas óptimos facilitan construir series de objetos 


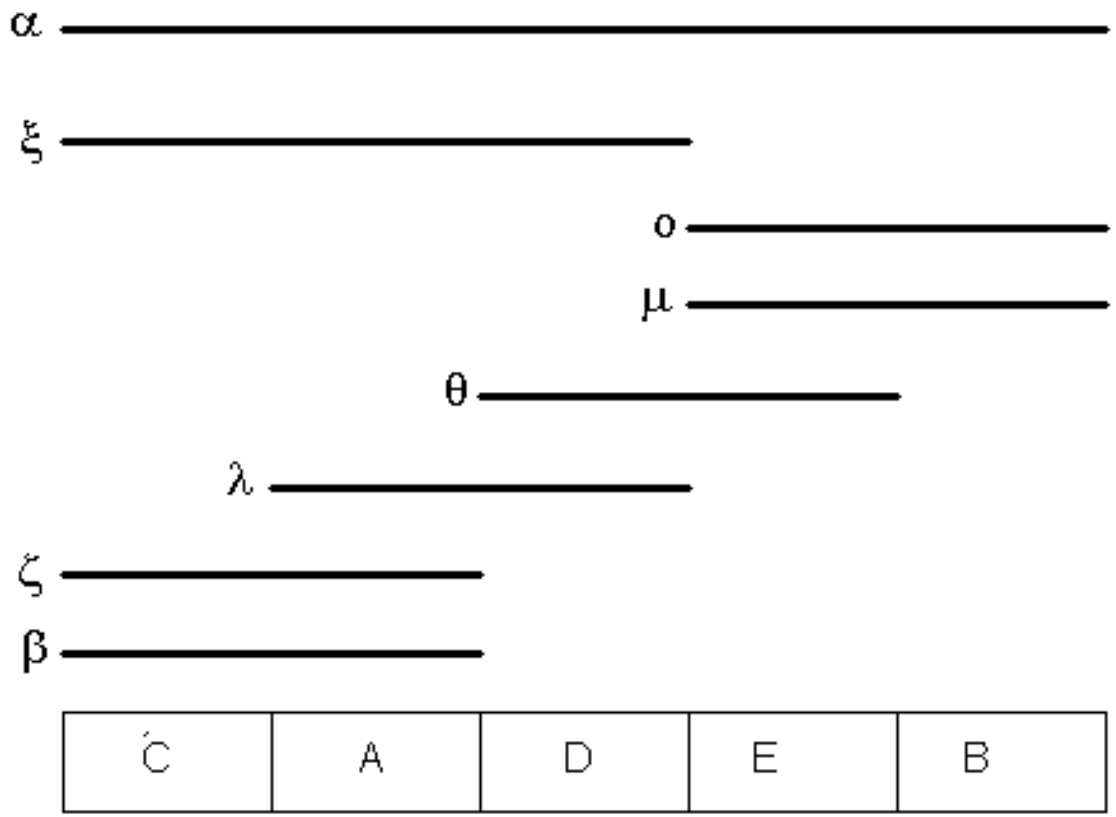

Figura 6. Recambio estructural que permiten inferir los retículos de las figuras 4 y 5 (sólo se muestran atributos comunes a dos o más objetos)

que delatan tendencias graduales de recambio de atributos. Si alteráramos la secuencia de objetos en la figura 6 , si en lugar de $\mathrm{C}-\mathrm{A}-\mathrm{D}-\mathrm{E}-\mathrm{B}$ pusiéramos $\mathrm{A}-\mathrm{C}-\mathrm{D}-\mathrm{E}-\mathrm{B}$ u otra disposición, generaríamos interrupciones en algunas de la líneas representativas de atributos comunes a más de un objeto (en nuestro ejemplo, cualquier otra serie, salvo B-E-D-A-C, da lugar a dichos cortes). Sin duda podríamos seguir hablando de una variación estructural a lo largo de la secuencia, pero la tendencia sería, de alguna manera, más errática. ¿Acaso importa? Tratándose del ordenamiento de teorías, es deseable un arreglo que ponga de relieve tendencias interpretables de variación estructural, esto es, que permitan vislumbrar no sólo la estructura del dominio, sino también cuestiones significativas del cambio teórico al interior de éste. A modo de regla heurística, nos parece que entre más gradual es la tasa de recambio estructural, seguramente es más expresiva de asuntos meta-científicos relevantes. De esto hablaremos con mayor detalle al tratar las teorías de la herencia. 


\section{Retículos para el dominio de la herencia}

En aras de construir, a modo de retículo, el dominio de la herencia, estipulamos que nuestros objetos son los elementos teóricos indicados en la tabla 1, cuyos atributos son las funciones y relaciones que figuran en la estructura de los modelos potenciales del elemento en cuestión; el apéndice 1 destaca los elementos y sus respectivas funciones y relaciones. ${ }^{6}$ Todos los elementos teóricos considerados incluyen partes relativas a la descendencia y al modo de concebir qué cosa es una característica heredable. Salvo la ley de herencia ancestral de Pearson (P), todos se montan sobre algún esquema tocante a la fisiología de la reproducción; y nuevamente con la excepción de Pearson, todos introducen factores, trasmisibles de progenitores a vástagos, que determinan los rasgos o características de los individuos. ${ }^{7}$ Por tanto, hemos decidido elaborar dos retículos: uno concerniente a las estructuras de la descendencia, reproducción y rasgos heredables (figura 7), y otro concerniente a estructuras que tipifican el comportamiento de factores hereditarios y, en el caso de Pearson, que tipifican correlaciones y fisonomías esperadas (figura 8). ${ }^{8}$ A grandes trazos, la figura 7 se refiere a las bases sobre las cuales teorizan las diversas propuestas, es decir, a las estructuras de los modelos potenciales parciales, mientras que el retículo de la figura 8 abarca las porciones teóricas de dichos esquemas. ${ }^{9}$

${ }^{6} \mathrm{El}$ apéndice es extenso y en aras de no extenderlo más hemos convenido en limitar la comparación entre elementos teóricos sólo con base en las funciones que figuran en las respectivas estructuras correspondientes a los modelos potenciales. Puesto que a cada función le corresponde un dominio y un codominio, los conjuntos primitivos (más otros que se derivan de éstos, mediante definición) de cada elemento teórico también están presentes en el apéndice, aunque ocultos. Podría objetarse el haber privilegiado la estructura de los modelos potenciales en lugar de los actuales; la razón de ello es que el formalismo correspondiente a los modelos actuales no introduce funciones nuevas, simplemente indica cómo es que se articula el ensamble conceptual (indicación que viene a ser la ley fundamental del elemento en cuestión).

${ }^{7}$ En cuanto a estos factores, los distintos autores propusieron diversos nombres, entre ellos: alelomorfo, bióforo, elemento, gémula, pangen. La diferenciación no estriba tanto en la nomenclatura, sino en las relaciones que dichos factores guardan con el resto de componentes del armazón conceptual. Por ejemplo, Brooks, Darwin y Galton hablan de gémulas, pero el término no tiene exactamente el mismo significado en la prosa de los tres investigadores.

${ }^{8}$ El procedimiento empleado para construir los retículos es el de Davey \& Priestley (2002), p.76-79.

${ }^{9}$ Cabe señalar que en el caso de la herencia ancestral de Pearson es muy discutible si acaso ésta introduce términos "herencia ancestral-teóricos", pues finalmente señala que los rasgos esperados de un individuo se derivan de una combinación algebraica de los rasgos 


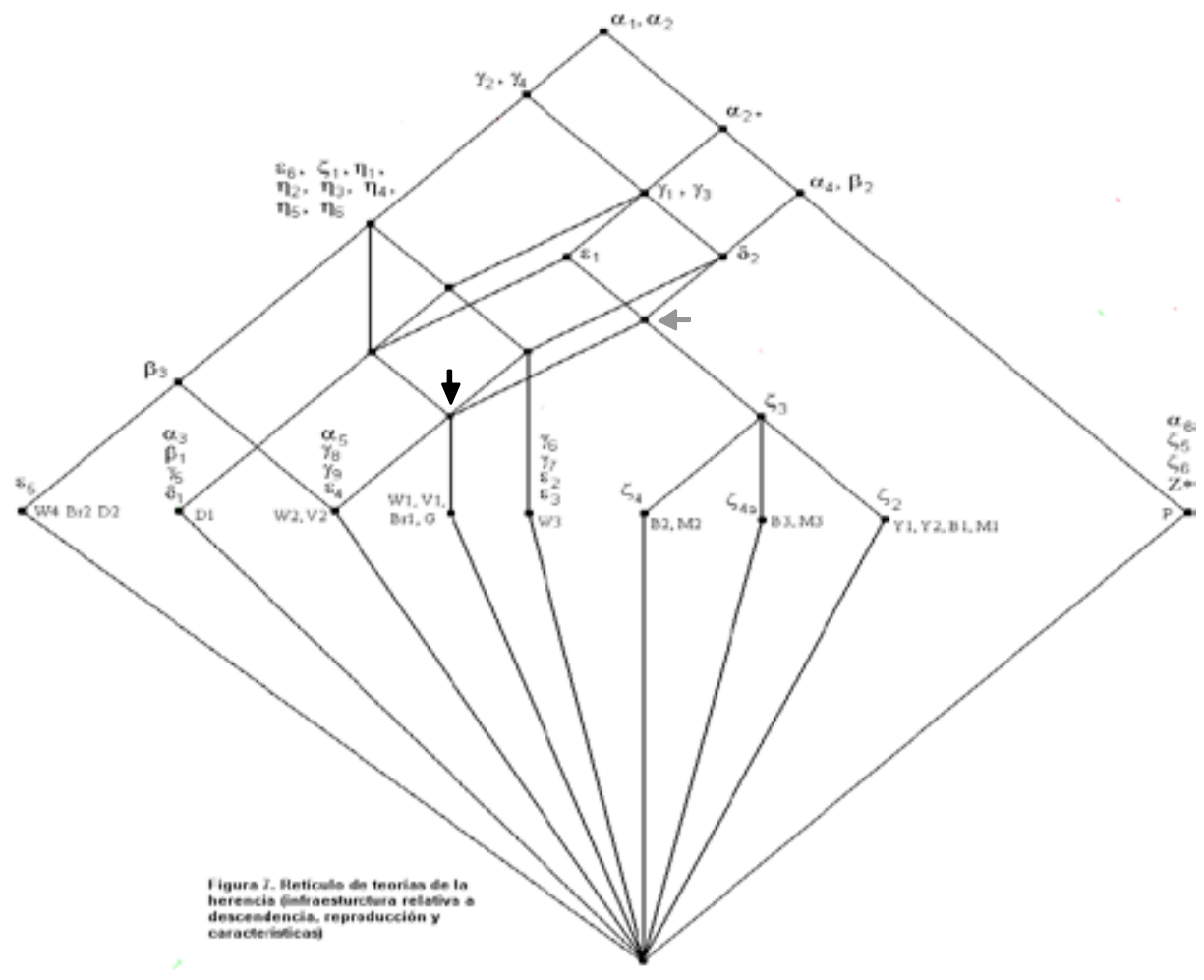

Al comparar ambos arreglos, destaca el hecho de que el diagrama de la figura 7 es menos distendido, por decirlo de algún modo, que el de la figura 8. Si uno se fija en los nodos de la parte central del "diamante" - la anticadena conformada por los llamados "conceptos-objeto"— en la figura 7 sólo hay tres que se corresponden con un único elemento teórico (los nodos rotulados D1, W3 y P), los demás albergan dos, tres o cuatro elementos. Esto es así, pues aquellos elementos teóricos que se reúnen en un mismo concepto-objeto presentan la misma estructura en lo concerniente a descendencia, reproducción y modos de concebir la noción de característica. La figura 8,

de sus ancestros, las correlaciones de cada atributo entre la población de descendientes y las múltiples poblaciones de ancestros (poblaciones de madres, padres, abuelos maternos, etc.) y las desviaciones estándares de cada rasgo en cada una de las poblaciones involucradas. Todo el aparato que Pearson monta sobre las relaciones de descendencia consiste en parámetros de distribuciones estadísticas idealizadas, y tales parámetros se estiman a partir de medidas tomadas a los individuos examinados. 


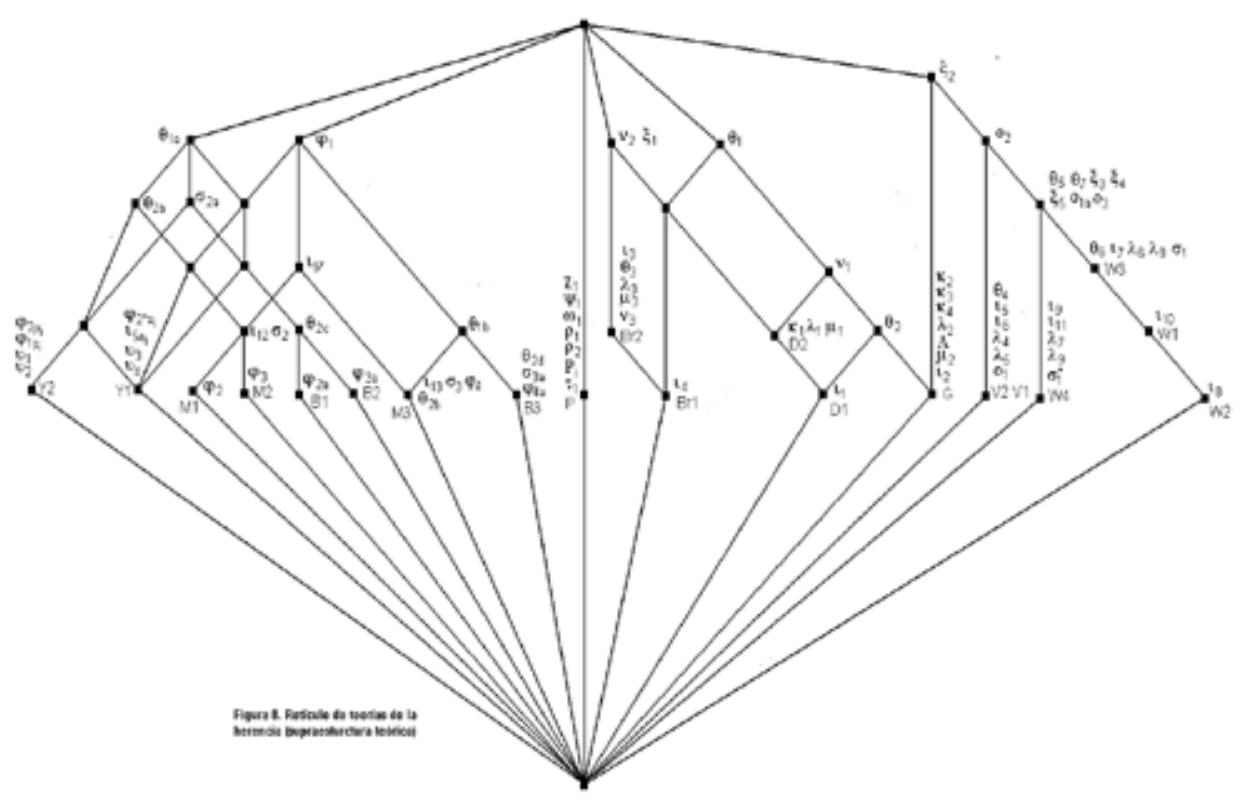

en cambio, presenta un despliegue de 19 conceptos-objeto, 14 de los cuales constan de un solo elemento teórico. El hecho de que haya conceptosobjeto que incluyan a más de un esquema teórico (los nodos rotulados $\mathrm{Br} 2$, $\mathrm{D} 2, \mathrm{~V} 1, \mathrm{~V} 2, \mathrm{~W} 1$ y W3) pudiera parecer extraño, pues no se distinguen entre sí, en lo tocante al comportamiento de factores hereditarios. En todos estos casos, la distinción estriba en la infraestructura relativa a la reproducción (como delata la figura 7); estos esquemas fueron pensados por sus creadores de tal modo que una parte medular de la estructura relativa al comportamiento de factores hereditarios se insta en diversos modos de reproducción. Nótese, por ejemplo, que toda la estructura teórica de $\mathrm{Br} 2$ está incluida en Br1, pero no viceversa. ${ }^{10}$ En fin, al contrastar las figuras 7 y 8 destaca que,

${ }^{10} \mathrm{Br} 1$ y $\mathrm{Br} 2$ son dos modalidades de la teoría de Brooks: el primero se refiere a organismos con reproducción sexual, mediante la fecundación unigamética; el segundo alude a organismos que se reproducen por partenogénesis. Ahora bien, Brooks consideraba que los óvulos portaban elementos — no les brinda otro nombre- que determinaban los rasgos constantes de la especie; por su parte, los gametos masculinos portan gémulas, las cuales inducen la variabilidad. Durante la fecundación, las gémulas "penetran" en los elementos del óvulo y les ocasionan un cambio de conformación, lo cual afecta la expresión de ese elemento en tanto que si antes determinaba una característica $\mathrm{X}$, ahora determina un carácter $\mathrm{X}^{*}$, 
entre 1865 y 1902, el dominio de la herencia —o por lo menos, la muestra que hemos tomado de ese dominio- acusaba una proliferación de supraestructuras teóricas, montadas sobre una base menos diversa de concepciones relativas a la descendencia, reproducción y caracteres.

Además de los conceptos-objetos, los demás nodos de cada retículo también representan concepciones de la época. Conviene abundar en sus significados, pero en aras de no hacer demasiado tediosa la presentación, sólo nos centraremos en unos cuantos. En la figura 7, la clase "teorías de la herencia que presuponen la fecundación unigamética y hacen hincapié en que las características se corresponden con mosaicos celulares" (designémosla FU-CMC para abreviar) está representada por el nodo que se indica con una flecha negra. Por tanto, esta clase abarca el conjunto de elementos teóricos $\{\mathrm{Br} 1, \mathrm{G}, \mathrm{V} 1, \mathrm{~V} 2, \mathrm{~W} 1, \mathrm{~W} 2\}$. Ahora bien, el hecho de que el nodo apuntado realmente se corresponde con ese tipo de fecundación y aquel modo particular de tratar los rasgos heredados, queda de manifiesto por los caminos que parten de allí y corren hacia arriba. Dos de ellos desembocan en el punto marcado por las funciones $\varepsilon_{6}, \zeta_{1}, \ldots, \eta_{6}$, las cuales aparecen en la figura 2 , misma que esquematiza la noción de caracteres como mosaicos celulares. Por otra parte, del nodo flechado en negro surge un trazo que llega a un punto adyacente a una saeta gris. Este punto se corresponde con la fecundación unigamética, pues los caminos que ascienden de allí pasan por nodos que ostentan todas las funciones constitutivas de la estructura de semejante fecundación, como puede apreciarse en la figura 1 (ciertas relaciones sobre la descendencia no se ilustran en la figura 1, pero constituyen la base sobre la cual se finca la fecundación unigamética).

Nótese ahora, también en la figura 7, el objeto-concepto titulado D1: como podrá verse, no hay ningún camino ascendente que parta de allí y llegue al punto representativo de la fecundación unigamética. Sin embargo, algunos de estos senderos sí pasan por nodos que sustentan funciones

ligeramente distinto a X. Por otra parte, Brooks señala que los tejidos vivos, sean de individuos masculinos o femeninos, producen gémulas ante cambios marcados en las condiciones de vida. En los machos, estas partículas migran a las células sexuales y se conglomeran allí. En el caso de las hembras, algunas gémulas que ellas misma producen pueden encontrar su camino a los óvulos y alterar los elementos allí contenidos; sin embargo, esto es un tanto accidental: el proceso fundamental por el cual gémulas entran al óvulo es la fecundación. Es menester subrayar que semejante dinámica de factores hereditarios se preserva en organismos partenogénicos, salvo lo concerniente al agrupamiento de gémulas en los gametos masculinos. Por eso, la supraestructura teórica de $\mathrm{Br} 2$ forma parte de la supraestructura teórica de Br1. 
constitutivas de esa reproducción, como son la producción de gametos y otras cuestiones. D1 se corresponde con la pangénesis darwiniana para organismos con reproducción sexual, pero Darwin consideraba que varios gametos masculinos (no uno solo) fertilizan un óvulo. Esto incluso tiene implicaciones sobre el modo en que Darwin concebía la paternidad, pues según su enfoque podría darse el caso de que un individuo fuera hijo de varios padres. Por eso, los caminos ascendentes con origen en D1 tampoco pasan por el punto que ostenta la función $\delta_{2}$, pues ésta asigna pares de gametos - uno masculino y otro femenino- a cigotos, en el entendido de que los miembros de cada par se funden para dar lugar a un cigoto. D1 se compone de otra función, $\delta_{1}$, que igual asigna pares de gametos a cigotos, pero uno de los miembros del par es un conjunto de gametos masculinos.

Entre otras cuestiones referentes a la figura 7, vale la pena mencionar que el nodo rotulado $\mathrm{W} 4, \mathrm{Br} 2, \mathrm{D} 2$ se corresponde con propuestas concernientes a la reproducción por partenogénesis $\left(\varepsilon_{5}\right.$ plantea que los organismos provienen de óvulos no fecundados). El concepto "teorías de la herencia que definen características heredables como características de líneas de descendencia cerrada” está representado por el punto marcado con $\xi_{3}$-función que a toda cepa pura le asigna una característica o una serie de características-, y aquí figuran todos los elementos teóricos de corte mendeliano. ${ }^{11}$ Por su parte, la idea de que los rasgos heredables son cuantificables se remite a la herencia ancestral de Pearson, nodo resaltado con el rótulo P. Finalmente, el retículo deja entrever una serie de recambio estructural que transita desde elementos teóricos que hacen hincapié en el basamento celular de la herencia, pero admiten diversos modos de reproducción; pasa por elementos teóricos que se fincan en un tipo de fecundación y un método particular para identificar rasgos heredables - la construcción de cepas puras- y termina en una propuesta que reduce las características a medidas, supone la reproducción sexual —en tanto se aplica a organismos que tienen un progenitor masculino y otro femenino-, pero no abunda sobre la fisiología reproductiva. Nos parece que la serie deja atisbar distintos modos de intervención experimental: todos aquellos elementos que enfatizan los mosaicos celulares hacen hincapié, —o bien, deberían hacerlo-, en técnicas de observación microscópica y corte histológico; los que ponen el acento en la construcción de cepas puras orientan los protocolos experimentales

${ }^{11} \mathrm{Al}$ usar expresiones como "elementos teóricos de corte mendeliano" o "elementos filomendelianos" y otras por el estilo, no estamos indicando que los elementos pertenecen a la misma red teórica. El mendelismo de Mendel y el de Bateson constituyen dos redes distintas, aunque muy parecidas. 
hacia rutinas de cruzamientos selectivos; y la propuesta de Pearson sugiere la confección de dispositivos y escalas para medir características y registrar los resultados.

Antes de entrar en los pormenores de la figura 8, conviene aclarar que los esquemas que suponen la existencia de factores hereditarios, también suponen que dichos factores se agrupan en clases o tipos funcionales, aunque la definición de tales categorías difiere, según el elemento teórico a considerar. ${ }^{12}$ Dirijamos nuestra atención ahora sobre los siguientes nodos de la figura 8:

- $\theta_{1 a}$. Este vértice podría describirse como la clase de teorías de la herencia que conciben a los gametos como portadores de series factoriales (una serie por gameto, y toda serie conformada por un factor de cada clase). En efecto, el nodo está rotulado por la función que asigna células sexuales a dichas fórmulas de factores. Si se siguen todos los caminos hacia abajo, queda claro que este concepto tiene por extensión el conjunto $\{\mathrm{B} 1, \mathrm{~B} 2, \mathrm{M} 1, \mathrm{M} 2, \mathrm{Y} 1, \mathrm{Y} 2\}$, conformado por las propuestas de filiación mendeliana salvo dos, M3 y B3, los esquemas de Mendel y Bateson, respectivamente, concernientes a la herencia con base en factores complejos (unidades compuestas de varios factores). En estos dos casos, las fórmulas gaméticas se componen de series de factores donde la i-ésima clase factorial puede estar representada por un integrante de ella o por una serie de factores de esa clase. La función $\theta_{1 \mathrm{~b}}$ opera la asignación correspondiente, y también es el rótulo de la clase de teorías que incluye solamente a M3 y B3.

- $\varphi_{1}$. El nodo se corresponde con la clase de teorías que suponen una relación directa, uno a uno, entre factores hereditarios y características (el factor $\mathrm{x}$ determina la característica específica y). Nuevamente, este concepto tiene por extensión casi todos los elementos teóricos allegados al mendelismo; Y2 es una excepción, pues esta propuesta de Yule establece una relación probabilística entre factores y caracteres, de tal suerte que el factor $X$ determina el rasgo $Y$ con probabilidad $Z$ y el carácter $\mathrm{Y}^{*}$ con probabilidad $\mathrm{Z}^{*}$.

${ }^{12}$ Los elementos teóricos de filiación mendeliana, por ejemplo, suponen clases de factores en correspondencia con tipos de características (v.g. la clase de factores que determinan la forma de la semilla del guisante); las distintas versiones de la pangénesis suponen que la i-ésima clase de factores se corresponde con el i-ésimo tipo de células (cardíacas, hepáticas, etc.); de Vries plantea que los factores integrantes de una determinada clase acusan el mismo tipo de arreglo molecular (puede haber variaciones, pero el "esqueleto" molecular es el mismo); Weismann sugiere algo similar a de Vries, pero sostiene, además, que una clase de factores determina subestructuras celulares - lo que hoy llamaríamos "organelos" - de un mismo tipo. 
- $v_{2} \xi_{1}$. Este punto delata la clase de teorías donde las células producen factores hereditarios y dichos factores migran a los gametos. El concepto agrupa las propuestas de Brooks y de Darwin, relativas a la pangénesis. La función $\xi_{1}$ vincula tríadas de células-actividadesambientes con series de gémulas, en tanto que la célula produce gémulas, pero tal producción está influida por las actividades que realiza la célula y las condiciones del medio en que vive: si su situación vital cambia, la célula producirá gémulas alteradas. Por su parte, $v_{2}$ asigna factores producidos a gametos, en tanto que ahí es adónde van los primeros. ${ }^{13}$ La formulación galtoniana de la pangénesis no figura aquí, pues Galton no le adjudica la producción gemular a las células, ni supone que las gémulas migran por el cuerpo. ${ }^{14}$

- $\theta_{1}$. La clase representada aquí es la de teorías de la pangénesis donde los gametos portan conjuntos de gémulas y al menos una gémula de cada tipo está representada. Figuran los dos esquemas darwinianos, el de Galton y uno de Brooks, pero en este último la función $\theta_{1}$ se restringe a gametos masculinos. La propuesta de Brooks tocante a la partenogénesis, $\mathrm{Br} 2$, no está incluida, pues el óvulo alberga factores hereditarios de naturaleza distinta a las gémulas (ver nota 10).

- $\mathrm{o}_{2}$. Este nodo pone de relieve la clase de teorías que destacan la importancia del núcleo celular en la herencia, pues allí está contenida la materia germinal. Quedan reunidos aquí los esquemas de Weismann y de Vries, aunque la organización interna del núcleo difiere en las distintas propuestas, cosa que se especifica en los ramales descendentes que surgen del nodo $\mathrm{O}_{2}$.

Se puede exprimir mucho más jugo del diagrama 8 , pero nos extenderíamos demasiado. De cualquier manera, merece comentario la tendencia general de variación estructural que deja entrever el arreglo, visto de izquierda a derecha. Si se omite el nodo P, la disposición transita de esquemas

${ }^{13}$ Dicho sea de paso, tanto en los esquemas darwinianos como en los de Brooks, las gémulas coadyuvan a determinar la fisonomía del individuo, pero lo hacen de manera distinta. Para Darwin, intervienen en la diferenciación celular, pues penetran células nacientes y les imprimen una forma y función. En cambio Brooks supone que entran en contacto con entidades generadoras de células (entidades contenidas en el óvulo) y alteran la configuración de ellas, lo que induce la génesis de células variantes (ver nota 10).

${ }_{14}$ Aunque no lo plantea de manera explícita, Galton (1875) sugiere que el cigoto es una especie de saco de gémulas, que va liberando estas entidades en el curso de la ontogenia. Las gémulas se convierten en células, y conforme se desarrolla la configuración celular del organismo, el cigoto va siendo "empujado" hacia el tejido reproductivo. Por otra parte, el stock de gémulas se reabastece, pues éstas tienen la capacidad de multiplicarse. 
que suponen una relación directa entre factores hereditarios y caracteres a elaboraciones que plantean una creciente mediación entre el factor y el rasgo fisonómico: las variantes de la pangénesis ( $\mathrm{Br} 2, \mathrm{Br} 1, \mathrm{D} 2, \mathrm{D} 1$ y G) establecen la secuencia factor-célula-característica, mientras que las teorías de de Vries y Weismann sostienen la serie factor-subestructura celular-célulacaracterística.

Vale la pena una última nota sobre los retículos de las figuras 7 y 8 : si uno contempla las estructuras de los elementos teóricos en formato de grafo (como se presenta en las figuras 1 a 3 para el caso V1) y toma en cuenta mapeos universales como la inclusión y la proyección, los nodos de ambos retículos revelan subestructuras conexas, esto es, funciones solas o composiciones de funciones. $\mathrm{Al}$ recorrer los caminos descendentes, desde el vértice superior hasta un cierto concepto-objeto, si uno traza las funciones que aparecen en los nodos intermedios - y las traza en otra hoja de papel, a manera de flechas, señalando dominios y codominios- irá conectando flechas hasta construir la estructura grafo del elemento teórico aludido por el concepto-objeto destino. Construye la base del elemento, si trabaja con la figura 7 , y la supraestructura teórica del mismo, si emplea la figura 8.

\section{Conclusiones}

En trabajos previos (Casanueva y Méndez, 2012(a) y 2012(b)) señalamos que los dominios teóricos constituyen espacios de confluencia de diversas teorías que tratan un problema común, aunque no necesariamente lo caracterizan de la misma manera. Argumentamos que la representación idónea de tales dominios es una constelación n-dimensional de puntos, donde cada uno de ellos representa un cierto elemento teórico, y las cercanías o lejanías entre puntos están en función de las similitudes estructurales de los elementos en cuestión. La idea detrás de semejante propuesta es que la forma de la configuración revela patrones significativos del dominio, tendencias que articulan las discusiones científicas involucradas. De hecho, recientemente hemos elaborado una representación así, en lo tocante a la herencia durante el periodo 1865-1902, como se muestra en la figura 9.

A pesar de que el enfoque nos sigue pareciendo atractivo, tiene problemas. La forma del dominio es un tanto caprichosa, pues depende del índice de similitud (o disimilitud) a usar, y depende también del algoritmo ordenador a emplear. Confeccionar la constelación no es cosa de "buen olfato"; se requiere el auxilio de programas de cómputo especializados. Existe un alud de técnicas multivariadas, generadoras de constelaciones, pero no ne- 


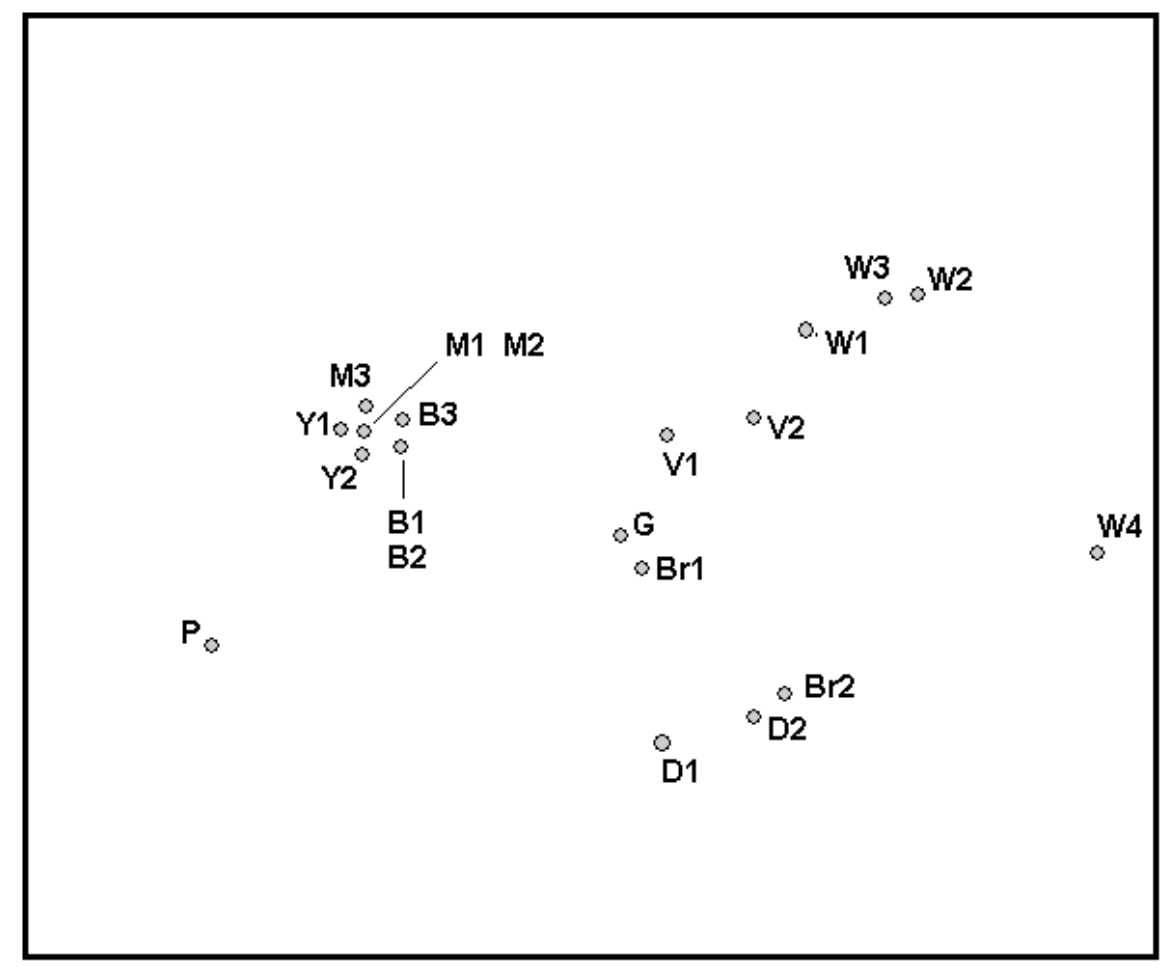

Figura 9. Ordenamiento de teorías de la herencia con el método de Kruskal

cesariamente brindan el mismo resultado, ni son buenas para toda ocasión. Por otra parte, hay un efecto de "caja negra", pues si bien el usuario puede conocer bien la teoría en que se sustenta el procedimiento, no tiene acceso a las operaciones del programa. Para elaborar la figura 9, los elementos teóricos se representaron como conjuntos de funciones, se calculó la cardinalidad de la diferencia simétrica para todos los pares de conjuntos, a manera de función de distancia interteórica; estos valores se sometieron a la rutina de escalamiento multidimensional que ofrece el programa SYSTAT 10, la cual se sustenta en el procedimiento desarrollado por Kruskal (1962 a y b).

Si bien la figura 9 ofrece una imagen interesante y, en efecto, la disposición de puntos sugiere tendencias de variación a estudiar, es mucho menos clara y explícita que los retículos antes expuestos. ${ }^{15}$ Más aún, éstos facilitan

${ }^{15}$ De cualquier manera, la figura 9 sí destaca la aglomeración de los elementos de inspiración mendeliana con mayor nitidez que los retículos 7 y 8 . Con todas las debidas reservas, se antoja pensar que esta aglomeración expresa de manera gráfica un caso de lo que Moulines denomina "cristalización". 


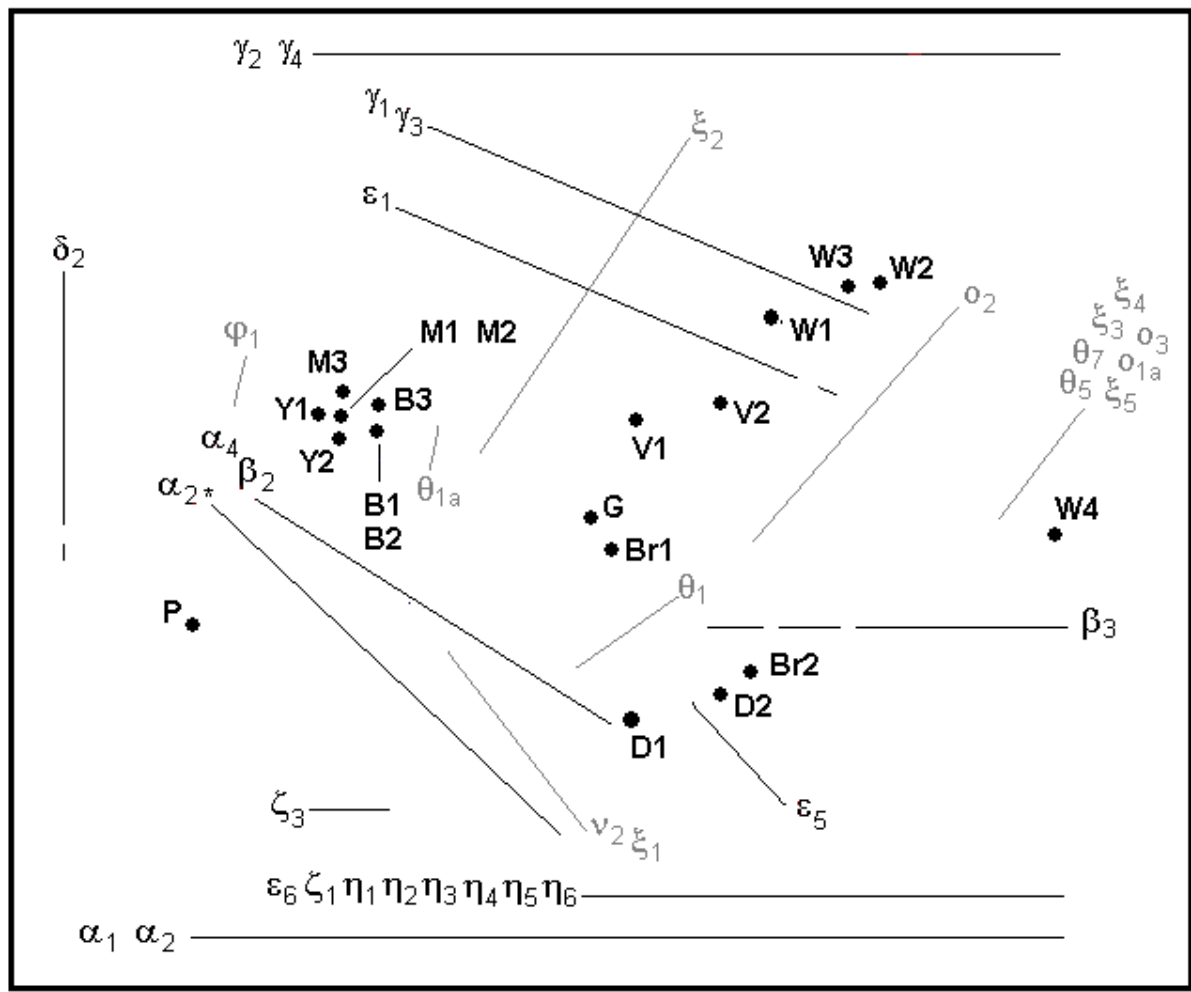

Figura 10. Configuración del dominio de la herencia con subestructuras sobrepuestas. En negro se indican las subestructuras relativas a la descendencia, reproducción y caracteristicas; en gris se resaltan las subestructuras tocantes al comportamiento de factores hereditarios. El barrido de las líneas pone de relieve qué subestructuras se corresponden con qué elementos teóricos.

la interpretación de los patrones de la figura, y para construirlos no fue menester recurrir a cajas negras computacionales. Para ilustrar cómo es que los retículos 7 y 8 nos pueden ayudar a entender la nube de puntos de la figura 9, sobre ella hemos puesto la información que dichos retículos nos brindan en cuanto a las presencias de algunas funciones en relación a los elementos teóricos considerados. El arreglo resultante se expone en la figura 10. Aquí, los segmentos representativos de funciones están dispuestos de tal manera que el "barrido" de ellos — a ambas lados- incluye solamente a aquellos elementos teóricos que ostentan la función de la cual se trata. En negro hemos representado las funciones propias de la descendencia, reproducción y modo de concebir características (destacadas en el retículo 7), y 
en gris representamos las concernientes a la organización y comportamiento de factores hereditarios. En fin, los retículos permiten recuperar un orden topológico para los dominios teóricos, lo cual puede aportar precisión a la noción de grado de variación conceptual.

En las primeras cuartillas de este escrito, sostenemos que la colección de teorías de la herencia de finales del siglo XIX y principios del XX ejemplifica un tipo de holón particular, que hemos denominado "dominio teórico". Cabe subrayar que los retículos elaborados aquí sólo son instrumentos para visualizar la variabilidad estructural al interior de un conjunto de teorías, pero no pretendemos decir que los holones o cualquier otra categoría metateórica del estructuralismo son retículos o debería representarse como retículos. No estamos autorizados para ello, pues lo único que hemos hecho es ilustrar patrones de variabilidad composicional que surcan a 20 elementos teóricos, pero no hemos construido armazones de relaciones interteóricas. Lo que sí afirmamos es que una buena imagen de la variación conceptual sirve para inferir asuntos importantes en lo que se refiere a las redes de relaciones interteóricas. En este sentido, la mancuerna dominio-retículo resulta una poderosa herramienta para explorar las complejas diversidades conceptuales propias tanto de la ciencia "inmadura" como de algunos episodios de la ciencia "madura", por decirlo en la terminología kuhniana.

Si bien el ejercicio realizado en el presente trabajo está acotado a un episodio científico particular, consideramos que de él se desprenden algunos lineamientos generales con respecto a los dominios teóricos. Señalar que tales entidades giran en derredor a una problemática merece mayor elucidación. El dominio se nos presenta como un cuerpo de ensambles conceptuales, donde unos cuantos sirven de parapeto a los demás, en tanto que estos últimos teorizan sobre los primeros. El dominio despliega series de teorizaciones no todas en paralelo, sino cruzadas, donde una estructura constituye la base de la cual se desprenden varias cadenas de teorizaciones sucesivas. En este sentido, el dominio teórico es un holón, pero un holón particular que privilegia vínculos teorizadores.

\section{Referencias bibliográficas}

Balzer, W., Moulines, C. U. \& J. Sneed, (1987) An Architectonic for Science. The Structuralist Program. Dordrecht: D. Reidel Publishing Company. Bateson, W. (1902) Mendel's Principals of Heredity. A Defense. London: Cambridge University Press. [edición facsimilar electrónica en: http:// www.esp.org/books/bateson/mendel/facsimile/] 
Bateson, W. (1979) Problems of Genetics. New Haven: Yale University Press.

Bateson, W. \& E.R. Saunders (1902) "The Facts of Heredity in the Light of Mendel's Discovery", Reports to the Evolution Committee of the Royal Society, I, pp. 125-160. [edición electrónica en: http://www.esp.org/ foundations/genetics/classical/holdings/b/wb-02b.pdf, pp. 1-39]

Brooks, W. K. (1883) The Law of Heredity. A Study of the Cause of Variation, and the Origin of Living Organisms. 2a edición, Baltimore: John Murphy. [Edición facsimilar electrónica en: http://www.esp.org/books/ brooks/heredity/facsimile/]

Casanueva, M. (2003) Mendeliana y anexos. México: Universidad Autónoma Metropolitana (Unidad Iztapalapa)-Miguel Ángel Porrúa, Colección Signos.

Casanueva, M. \& D. (2012): "Grafos representacionales y dinámica de teorías" en: José Luis Peris-Viñe (ed.), Filosofía de la Ciencia en Iberoamérica: Metateoría Estructural, Tecnos, Madrid pp. 485-505.

Casanueva, M. \& D. Méndez (2012): "Poblaciones de modelos y dinámicas científicas" en Stoa. Revista del Instituto de Filosofía de la Universidad Veracruzana Vol. 3, no. 5, pp. 159-179.

Darwin, C. (1883) The Variations of Animals and Plants Under Domestication. Volumes I and II. 2a edición, New York: Appleton. [edición facsimilar electrónica en http://www.esp.org/books/darwin/variation/ facsimile/]

Davey, B. A. \& Priestly, H. A. (2002). Introduction to Lattices and Order. Cambridge: Cambridge University Press.

de Vries, H. (1910) Intracellular Pangenesis, Including a Paper on Fertilization and Hybridization. Stuart Gager (trad.) Chicago: Open Court Publishing Co. [edición facsimilar electrónica: http://www.esp.org/books/ devries/pangenesis/facsimile/].

Díez, J.A. \& C.U. Moulines (1997). Fundamentos de la filosofía de la ciencia. Barcelona: Ariel.

Galton, F. (1875) “A Theory of Heredity”, Contemporary Review, 27: 8095.

Galton, F. (1997) Natural Inheritance, Gentetics Heritage Press: New Mexico. [Edición facsimilar del original publicado en 1889 por Macmilan and Co. (London)].

Gillham, N. W. (2001) A Life of Sir Francis Galton. From African Explorer to the Birth of Eugenics. Oxford: Oxford University Press.

Grätzer, G. (2000) General Lattice Theory. Basel: Birkhäuser Verlag. 
Kruskal, J (1964a) “Multidimensional Scaling by Optimizing Goodness-offit to a Nonmetric Hypothesis", Psychometrika, 29: 1-28.

Kruskal, J (1964b) "Non-metric Multidimensional Scaling: A Numerical Method", Psychometrika, 29: 115-129.

Lawvere, F.W. \& S. H. Schanuel (1997) Conceptual Mathematics. A First Introduction to Categories. Cambridge: Cambridge University Press.

Lorenzano, P (2000) "Classical Genetics and the Theory-Net of Genetics" en Poznan Studies in the Philosophy of the Sciences and the Humanities, vol. 75 (Wolfgang Balzer, Joseph D. Sneed \& C. Ulises Moulines (eds.) Structuralist Knowledge Representation: Paradigmatic Examples), pp. $251-283$.

Lorenzano, P.: "La emergencia de un progama de investigación en genética”, en: P. Lorenzano, L.A.-C.P. Martins y A. C. Regner (comps.), Ciências da vida: estudos filosóficos e históricos, Filosofia e História da Ciência no Cone Sul, vol. 2. Campinas, 2006.

Méndez, D. (2006) Paisajes conceptuales de la herencia biológica entre 1865 y 1902. Reconstrucción y ordenamiento de teorías de la herencia. Tesis para obtener el título de Doctor en Humanidades, Universidad Autónoma Metropolitana, Unidad Iztapalapa, México.

Mendel, G. (1866) "Versuche über Planzenhybriden" Verhandlungen des naturforschenden Vereines in Brünn, Bd. für das Jahr 1865, Abhandlungen, 3-47 ["Experiments in Plant Hybridization” en página electrónica: http://www.esp.org/foundations/genetics/classical/gm-65.pdf, pp. 1-39].

Moulines, C. U. (1982) Exploraciones metacientíficas. Estructura, desarrollo y contenido de la ciencia. Madrid: Alianza.

Moulines, C.U (2011), "Cuatro tipos de desarrollo teórico en las ciencias", Metatheoria 1(2): 11-27

Pearson, K. (1896) "Mathematical Contributions to the Theory of Evolution. III. Regression, Heredity, and Panmixia" en Philosophical Transactions of the Royal Society of London, ser. A, 187: 253-318.

Ross, K., Wright, C. (1990) Matematicas Discretas. México: Prentice-Hall Hispanoamericana.

Shapere, D. (1977) "Scientific Theories and Their Domains" en Frederick Suppe (ed) The Structure of Scientific Theories. Urbana: University of Illinois Press, pp. 518-565.

Weldon, W. F. R. (1901-1902) “Mendel's Laws of Alternative Inheritance in Peas", Biometrika 1: 228-254.

Weismann, A. (1893) The Germ-plasm. A Theory of Heredity. Newton Parker \& Harriet Rönnfeldt (trads.). New York: Charles Scribner's Sons. 
[edición facscimilar electrónica: http://www.esp.org/books/weismann/ germ-plasm/facsimile/]

Willie, R. (2005) "Formal Concept Analysis as Mathematical Theory of Concepts and Concept Hierarchies”, en Ganter, B., Stumme, G. \& Willie, R. (eds) Formal Concept Analysis: Foundations and Applications. Berlin: Springer-Verlag, pp. 1-33.

Yule, G.U. (1902) "Mendel's laws and their probable relations to intraracial heredity". New Phytologist, 1: 193-200, 222-238. 
APÉNDICE 1: ELEMENTOS TEÓRICOS Y FUNCIONES ASOCIADAS

\begin{tabular}{|c|c|c|c|c|c|c|c|c|c|c|c|c|c|c|c|c|c|c|c|c|c|}
\hline & & $\mathbf{P}$ & $\begin{array}{l}\mathrm{Y} \\
1 \\
\end{array}$ & $\begin{array}{l}\mathrm{Y} \\
2\end{array}$ & $\begin{array}{l}\mathrm{B} \\
3 \\
\end{array}$ & $\begin{array}{l}\mathrm{B} \\
2\end{array}$ & $\begin{array}{l}\text { B } \\
1 \\
\end{array}$ & $\begin{array}{c}\mathrm{M} \\
3\end{array}$ & $\begin{array}{c}M \\
2\end{array}$ & $\begin{array}{c}M \\
1\end{array}$ & $\begin{array}{l}\text { W } \\
3 \\
\end{array}$ & $\begin{array}{l}W \\
2\end{array}$ & $\begin{array}{l}\text { W } \\
4\end{array}$ & $\begin{array}{c}\mathrm{W} \\
1 \\
\end{array}$ & $\begin{array}{l}\mathrm{V} \\
2\end{array}$ & $\begin{array}{l}\mathrm{V} \\
1 \\
\end{array}$ & $\begin{array}{c}\mathrm{Br} \\
2 \\
\end{array}$ & \begin{tabular}{|c|}
$\mathrm{Br}$ \\
1 \\
\end{tabular} & $\mathrm{G}$ & $\begin{array}{l}\mathrm{D} \\
2 \\
\end{array}$ & \begin{tabular}{|c|}
$\mathrm{D}$ \\
1 \\
\end{tabular} \\
\hline 1 & $\alpha_{1}$ & $\mathrm{X}$ & $\mathrm{X}$ & $\mathrm{X}$ & $\mathrm{X}$ & $X$ & $X$ & $X$ & $X$ & $\mathrm{X}$ & $\mathrm{X}$ & $X$ & $X$ & $X$ & $\mathrm{X}$ & $X$ & X & $X$ & $\mathrm{X}$ & $\mathrm{X}$ & $X$ \\
\hline 2 & $\alpha_{2}$ & $\mathrm{X}$ & $\mathrm{X}$ & $\mathrm{X}$ & $\mathrm{X}$ & $\mathrm{X}$ & $\mathrm{X}$ & $\mathrm{X}$ & $\mathrm{X}$ & $X$ & $X$ & $X$ & $\mathrm{X}$ & $X$ & $X$ & $X$ & $\mathrm{X}$ & $X$ & $\mathrm{X}$ & $X$ & $X$ \\
\hline 3 & $\alpha_{2^{*}}$ & $\mathrm{X}$ & $\mathrm{X}$ & $\mathrm{X}$ & $\mathrm{X}$ & $\mathrm{X}$ & $\mathrm{X}$ & $\mathrm{X}$ & $\mathrm{X}$ & $\mathrm{X}$ & $\mathrm{X}$ & $\mathrm{X}$ & & $\mathrm{X}$ & $\mathrm{X}$ & $\mathrm{X}$ & & $X$ & $\mathrm{X}$ & & $X$ \\
\hline 4 & $s \alpha_{2}$ & $\mathrm{X}$ & $\mathrm{X}$ & $\mathrm{X}$ & $\mathrm{X}$ & $\mathrm{X}$ & $\mathrm{X}$ & $\mathrm{X}$ & $\mathrm{X}$ & $\mathrm{X}$ & $\mathrm{X}$ & $\mathrm{X}$ & & $\mathrm{X}$ & $\mathrm{X}$ & $\mathrm{X}$ & & $X$ & $\mathrm{X}$ & & $X$ \\
\hline 5 & $\alpha_{3}$ & & & & & & & & & & & & & & & & & & & & $X$ \\
\hline 6 & $\alpha_{4}$ & $\mathrm{X}$ & $\mathrm{X}$ & $\mathrm{X}$ & $\mathrm{X}$ & $\mathrm{X}$ & $\mathrm{X}$ & $\mathrm{X}$ & $\mathrm{X}$ & $\mathrm{X}$ & $\mathrm{X}$ & $\mathrm{X}$ & & $\mathrm{X}$ & $\mathrm{X}$ & $\mathrm{X}$ & & $X$ & $\mathrm{X}$ & & \\
\hline 7 & $\alpha_{5}$ & & & & & & & & & & & $\mathrm{X}$ & & & $\mathrm{X}$ & & & & & & \\
\hline 8 & $\alpha_{6}$ & $\mathrm{X}$ & & & & & & & & & & & & & & & & & & & \\
\hline 10 & $\beta_{1}$ & & & & & & & & & & & & & & & & & & & & $\mathrm{X}$ \\
\hline 12 & $\beta_{2}$ & $\mathrm{X}$ & $\mathrm{X}$ & $\mathrm{X}$ & $\mathrm{X}$ & $\mathrm{X}$ & $\mathrm{X}$ & $\mathrm{X}$ & $\mathrm{X}$ & $\mathrm{X}$ & $\mathrm{X}$ & $\mathrm{X}$ & & $\mathrm{X}$ & $\mathrm{X}$ & $\mathrm{X}$ & & $X$ & $\mathrm{X}$ & & \\
\hline 14 & $\beta_{3}$ & & & & & & & & & & & $X$ & $\mathrm{X}$ & & $X$ & & $\mathrm{X}$ & & & $\mathrm{X}$ & \\
\hline 16 & $\gamma_{1}$ & & $\mathrm{X}$ & $\mathrm{X}$ & $\mathrm{X}$ & $\mathrm{X}$ & $\mathrm{X}$ & $\mathrm{X}$ & $X$ & $\mathrm{X}$ & $\mathrm{X}$ & $X$ & & $\mathrm{X}$ & $\mathrm{X}$ & $\mathrm{X}$ & & $X$ & $\mathrm{X}$ & & $\mathrm{X}$ \\
\hline 17 & $\gamma_{2}$ & & $\mathrm{X}$ & $\mathrm{X}$ & $\mathrm{X}$ & $\mathrm{X}$ & $\mathrm{X}$ & $X$ & $\mathrm{X}$ & $\mathrm{X}$ & $\mathrm{X}$ & $\mathrm{X}$ & $X$ & $\mathrm{X}$ & $\mathrm{X}$ & $\mathrm{X}$ & $\mathrm{X}$ & $X$ & $\mathrm{X}$ & $\mathrm{X}$ & $\mathrm{X}$ \\
\hline 18 & $\gamma_{3}$ & & $\mathrm{X}$ & $\mathrm{X}$ & $\mathrm{X}$ & $\mathrm{X}$ & $\mathrm{X}$ & $X$ & $X$ & $X$ & $X$ & $X$ & & $X$ & $\mathrm{X}$ & $\mathrm{X}$ & & $X$ & $\mathrm{X}$ & & $X$ \\
\hline 19 & $\gamma_{4}$ & & $\mathrm{X}$ & $\mathrm{X}$ & $\mathrm{X}$ & $\mathrm{X}$ & $\mathrm{X}$ & $\mathrm{X}$ & $\mathrm{X}$ & $X$ & $\mathrm{X}$ & $\mathrm{X}$ & $\mathrm{X}$ & $X$ & $\mathrm{X}$ & $\mathrm{X}$ & $\mathrm{X}$ & $X$ & $\mathrm{X}$ & $\mathrm{X}$ & $X$ \\
\hline 20 & $\gamma_{5}$ & & & & & & & & & & & & & & & & & & & & $\mathrm{X}$ \\
\hline 21 & $\gamma_{6}$ & & & & & & & & & & $X$ & & & & & & & & & & \\
\hline 22 & $\gamma_{7}$ & & & & & & & & & & $\mathrm{X}$ & & & & & & & & & & \\
\hline 23 & $\gamma_{8}$ & & & & & & & & & & & $\mathrm{X}$ & & & $\mathrm{X}$ & & & & & & \\
\hline 24 & $\gamma_{9}$ & & & & & & & & & & & $\mathrm{X}$ & & & $\mathrm{X}$ & & & & & & \\
\hline 25 & $\delta_{1}$ & & & & & & & & & & & & & & & & & & & & $\mathrm{X}$ \\
\hline 26 & $\delta_{2}$ & & $\mathrm{X}$ & $\mathrm{X}$ & $\mathrm{X}$ & $\mathrm{X}$ & $\mathrm{X}$ & $\mathrm{X}$ & $\mathrm{X}$ & $X$ & $\mathrm{X}$ & $\mathrm{X}$ & & $\mathrm{X}$ & $\mathrm{X}$ & $\mathrm{X}$ & & $X$ & $\mathrm{X}$ & & \\
\hline 27 & $\varepsilon_{1}$ & & $\mathrm{X}$ & $\mathrm{X}$ & $\mathrm{X}$ & $\mathrm{X}$ & $\mathrm{X}$ & $\mathrm{X}$ & $\mathrm{X}$ & $\mathrm{X}$ & & $\mathrm{X}$ & & $\mathrm{X}$ & $\mathrm{X}$ & $\mathrm{X}$ & & $\mathrm{X}$ & $\mathrm{X}$ & & $\mathrm{X}$ \\
\hline 28 & $\varepsilon_{2}$ & & & & & & & & & & $\mathrm{X}$ & & & & & & & & & & \\
\hline 29 & $\varepsilon_{3}$ & & & & & & & & & & $\mathrm{X}$ & & & & & & & & & & \\
\hline
\end{tabular}

Nota: la tabla completa del apéndice 1 consta de 132 filas; en aras de economizar espacio sólo se presentan las primeras 29. La reconstrucción de los elementos teóricos mencionados en el presente artículo, así como la tabla completa de funciones versus elementos teóricos, se presentan —aunque con otra notación- en Méndez (2006), tesis que se puede consultar en la dirección electrónica: http://148.206.53.231/UAMI13466.pdf 